\title{
Solution, Extensions and Applications of the Schauder's 54th Problem in Scottish Book
}

\author{
Milan R. TASKOVIĆ*
}

\begin{abstract}
This paper presents the Axiom of Infinite Choice: Given any set $P$, there exist at least countable choice functions or there exist at least finite choice functions. The author continues herein with the further study of two papers of the Axiom of Choice in order by E. Zermelo [Neuer Beweis für die Möglichkeit einer Wohlordung, Math. Annalen, 65 (1908), 107-128; translated in van Heijenoort 1967, 183-198], and by M. Tasković [The axiom of choice, fixed point theorems, and inductive ordered sets, Proc. Amer. Math. Soc., 116 (1992), 897-904]. Fredholm and Leray-Schauder alternatives are two direct consequences of the Axiom of Infinite Choice! This paper presents applications of the Axiom of Infinite Choice to the Fredholm and Leray-Schauder theory. In this sense, I give a solution and some extensions of Schauder's problem (in Scottish book, problem 54). This paper presents some new mathematical $n$-person games. In the theory of $n$-person games, there have been some further developments in the direction of transversal games and mathematical alternative theory.
\end{abstract}

\section{History AND ORIGINS}

We shall first discuss an assumption that appears to be independent of, and yet consistent with, the usual logical assumptions regarding classes and correspondences, but whose absolute validity has been seriously questioned by many authors. This is the so-called Axiom of Choice, which has excited more controversy than any other axiom of set theory since its formulation by Ernst Zermelo in 1908. In this sense, many results are known in the set theory.

2000 Mathematics Subject Classification. Primary: 01A55, 01A60, 03-03, 03E25, 0403, 04A25. Secondary: 47H10, 05A15, 54H25.

Key words and phrases. The Axiom of Infinite Choice, Zermelo's Axiom of Choice, Lemma of Infinite Maximality, Zorn's lemma, Foundation of the Fixed Point Theory, Geometry of the Axiom of Infinite Choice, Axioms of Infinite Choice for Points and Apices, General Brouwer theorem, General Schauder theorem, Extension of Schauder's 54 th problem in Scottish book, Transversal $n$-person games, Nash's theorem, Peano's theorem. 
In 1904, Zermelo $^{1}$ stated a principle of choice similar to: If $\mathcal{D}$ is a family of nonempty sets, there is a function $f$ such that $f(A) \in A$ for every $A \in \mathcal{D}$; and proved that it implied the well-ordering theorem. In 1908 Zermelo proposed main version of the Axiom of Choice. This is the connection and with a conversations with Erhard Schmidt.

Bertrand Russell in 1906 gave a principle analogous to preceding. He announced this principle as a possible substitute for Zermelo's but he believed that it was weaker. Zermelo, in 1908 stated and, proved that Russell's and his formulations of the axiom of choice are equivalent. The name "axiom of choice" is due to Zermelo in 1904.

Apparently, the first specific reference to the axiom of choice was given in a paper by G. Peano ${ }^{2}$ in 1890 . In proving an existence theorem for ordinary differential equations, he ran across a situation in which such a statement is needed. In 1886 Peano published a new demonstration of the theorem, due to A. Cauchy, that the differential equation

$$
y^{\prime}=f(x, y), \quad y\left(x_{0}\right)=t_{0},
$$

has a unique solution. Here Peano weakened Cauchy's hypotheses to require only that $f(x, y)$ be continuous. Four years later Peano returned to this theorem and generalized his proof to finite systems of first-order equations.

Beppo Levi in 1902, while discussing the statement that the union of a disjoint set $S$ of nonempty sets has a cardinal number greater than or equal to the cardinal number of $S$, remarked that its proof depended on the possibility of selecting a single member from each element of $S$. Others, including Georg Cantor, had used the principle earlier, but did not mention it specifically.

In 1892 R. Bettazzi, who had just become Peano's colleague at the Military Academy in Turin, published an article on discontinuous real functions - with terminology of infinite many arbitrary choices.

In this time, the Axiom of Choice asserts that for every set $S$ there is a function $f$ which associates each nonempty subset $A$ of $S$ with a unique member $f(A)$ of $A$. From a psychological perspectie, one might express the Axiom by saying that on element is "chosen" from each subset $A$ of $S$. However, if $S$ is infinite, it is difficult to conceive how to make such choices - unless a rule is available to specify an element in each $A$.

\footnotetext{
${ }^{1}$ Before 1904, when Zermelo published his proof that the axiom of choice implies the well-ordering theorem, the well-ordering theorem was considered as self-evident. Cant or and the others used it without hesitation.

${ }^{2}$ Giuseppe Peano: "But as one cannot apply infinitely many times an arbitrary rule by which one assigns to a class $A$ an individual of this class, a determinate rule is stated here".
} 
David Hilbert, in 1926, once wrote that Zermelo's Axiom of Choice ${ }^{3}$ was the axiom "most attacked up to the present in the mathematical literature..."; to this, Abraham Fraenkel later added that "the axiom of choice is probably the most interesting and, in spite of its late appearance, the most discussed axiom of mathematics, second only to Euclid's axiom of parallels which was introduced more than two thousand years ago".

The equivalence of the axiom of choice and the trichotomy was given by Hartogs in 1915. As in the case of the well-ordering theorem, the trichotomy was considered self-evident and was used without hesitation before 1915 .

As mathematics developed futher there also developed a need for another non-constructive proposition; a principle, which Kuratowski, Hausdorff, Zorn, and others, used to replace transfinite induction and the well-ordering theorem. It appears, at first glance, unrelated to the axiom of choice, but actually is equivalent to it.

This principle and principles similar to it are often referred to as forms of Zorn's lemma. In 1933 Artin and Chevalley first referred to the principle as Zorn's lemma.

The history of maximal principles is quite tangled. The earliest reference to a maximal principle in the literature is in 1907 from Hausdorff.

In 1910 independently Janiszewski, Mazurkiewicz and Zoretti published a special case Hausdorff's principle in the form of a theorem in topology. In 1905 Lindelöf, in 1911 Brouwer, and in 1920 Sierpiński derivated some more general topological theorems from the well-ordering theorem.

In 1922 Kuratowski derived minimal principles equivalent to the preceding principles from the well-ordering theorem. Kuratowski in 1922 used a minimal principle to prove a theorem in analysis, as and R. L. Moore in 1932 .

In set theory, we notice that, all of the usual mathematical concepts can be reduced to the notion of set. ${ }^{4}$

\footnotetext{
3ZZermelo's Reply to His Critics. During the summer of 1907 Zermelo took stock of the criticisms directed against both his Axiom and his proof of the well-ordering theorem. One in 1908 was a reply to his critics, and the other also in 1908 contained the first axiomatization of set theory. Zermelo's first article in 1908 began with a new demonstration of the well-ordering theorem.

From them he developed the properties of his $\theta$-chains, which generalized Dedekind's earlier concept of chain. Zer melo corresponded with J ourdain in 1907, but apparently their letters focused on a generalization of König's theorem.

Although he had read Borel's article and the published correspondence between: Baire, Borel, Hadamard, and Lebesgue, he concetrated on refuting Peano with whom he had previously feuded over the equivalence theorem.

During 1906 he corresponded with Poincaré regarding his proof and his axiomatization of set theory. A letter, as well as three others from Poincaré, is kept in Zermelo's Nachlass at the University of Freiburg in Breisgau. De facto, Zermelo emerged as a realist in much situations, perhaps a Platonist!?

${ }^{4}$ Fixed Point Problem. I have to admit that the Axiom of Infinite Choice is the main source, i.e., to say the very beginning of a fixed point theory. It is an open question whether
} 
Within the Cantorian tradition, one can view Zermelo's axiomatization as answering the question: What is a set?! This question has served as a theme in the development of set theory, but one not often discussed openly.

In the meanthime, there has developed a concept of the set-theory damaged school child, so we must ensure that this paradise remains a blooming garden and does not turn into rocky ground and thorny scrub. In this sense, our admission, for this problem in this paper, give a new paradise for set-theory.

We notice that the Axiom of Infinite Choice is main spring (origin) for the fixed point theory. In this sense, the fixed point problem for a given mapping $f \mid P$ is very easy to formulate: the question is whether some $\xi \in P$ satisfies $f(\xi)=\xi$. Many problems are reducible to the existence of fixpoints of certain mappings. The question remains whether some statement (of the axiom of choice type) could be equivalently expressed in the fixpoint language as well. The answer is affirmative. In this sense, the equation for $x \in P$ in the following form

$$
\sup \{x, f(x)\}=x \quad\left(\text { or } \sup \left\{x, f^{2}(x)\right\}=x\right)
$$

for a given map $f \mid P$ and for a nonempty partially ordered set $P$ is a key object for new equivalents of the Axiom of Infinite Choice.

In this paper we prove some new equivalents of the Axiom of Infinite Choice in connection with (Eq). These statements are of fixed point type theorems and fixed apex type theorems. Applications in fixed point theory are considered.

Call a poset (=partially ordered set) $P$ inductive (chain complete) when every nonempty chain in $P$ has an upper bound (least upper bound, i.e., supremum) in $P$. Also, call a poset $P$ quasi-inductive (quasi-chain complete) when every nonempty well ordered chain has an upper bound (supremum) in $P$.

Also, we consider the concept of fixed apices for the mapping $f$ of a poset $P$ into itself. A map $f$ of a partially ordered set $P$ to itself has a fixed apex $u \in P$ if for $u \in P$ there is $v \in P$ such that $f(u)=v$ and $f(v)=u$.

an affirmation of the Axiom of Infinite Choice form might be presented equivalently by words, defining fixed points. The answer to this question is positive. In this sense, for $x \in P$ the equation of the form $x=f(x)$ or

$$
\sup \{x, f(x)\}=x(\text { or } \inf \{x, f(x)\}=x)
$$

for a given mapping $f \mid P$ and for a nonempty partially ordered set $P$ : is the key objects in this case, both for new equivalents of the Axiom of Infinite Choice and for further work on the Fixed point theory.

Otherwise, the first dynamic examples from the Fixed Point Theory go back to 1700 B.C-. the time when Moses, together with the Israelites, fleeing from Egypt, crossed the river Jordan, as well as from 650 B.C. when this river was crossed by St. Ilija and his disciple Jelisej; see: the Old Testament. 
Fixed points are clearly fixed apices and the set of all fixed points can be a proper subset of the set of fixed apices.

On the other hand, $f$ has a fixed apex if and only if $f^{2}:=f(f)$ has a fixed point. Indeed, for if $f$ has a fixed apex $u \in P$, then $u=f(v)$ and $v=f(u)$, so $f^{2}$ has a fixed point. If the equation $x=f^{2}(x)$ has a solution $\xi=f^{2}(\xi)$ for some $\xi \in P$, then $f$ has fixed apices $\xi, f(\xi) \in P$ because $\xi=f^{2}(\xi)$ and $f(\xi)=f(\xi)$.

An important class of sets is the class of countable sets. More precisely we say that a set $X$ is countable if and only if there is a one-to-one mapping of $\mathbb{N}(:=\{1,2, \ldots, n, \ldots\})$ onto $X$. A set which is not countable is said to be uncountable. ${ }^{5}$

\section{The Lemma of Infinite Maximality}

By the "Axiom of Infinte Choice" we mean a statement in the following form as: Given any set $S$, there exist at least countable choice functions or there exist at least finite choice functions. In this sense we prove some new equivalents of the Axiom of Infinite Choice. But perhaps the most statement equivalent to the Axiom of Infinite Choice is the following statement.

Theorem 2.1. (Lemma of Infinite Maximality). Let $P$ be an inductiuve partially ordered set with ordering $\preccurlyeq$, then $P$ has at least countable maximal elements or $P$ has at least finite maximal elements.

Proof. (Application of the Axiom of Infinite Choice). Let card $P=m$ and card $B(m)=\alpha$, where $B(m)$ is denoted the set of all ordinal numbers $\alpha$ such that $\bar{\alpha} \leq m$. For an indirect proof suppose that for every $y \succcurlyeq x$ the set $\{z \in P: y \prec z\}$ is nonempty. Define the transfinite sequences $\left\{y_{\beta}^{k}\right\}_{\beta<\alpha}$ for $k \in \mathbb{N}$ in the following form as

$$
y_{\beta}^{k}=\left\{\begin{array}{l}
\text { the upper bound of }\left\{x_{\gamma}^{k}\right\}_{\gamma<\beta}, \text { if it exist }, \\
x \text { otherwise }
\end{array}\right.
$$

for $k \in \mathbb{N}$ and define the transfinte sequences $\left\{x_{\beta}^{k}\right\}_{\beta<\alpha}$ by

$$
x_{\beta}^{k}=f_{k}\left(\left\{z \in X: y_{\beta}^{k} \prec z\right\}\right), \quad \text { for } k \in \mathbb{N},
$$

where $f_{k}:(\mathcal{P}(P) \backslash\{\varnothing\}) \rightarrow P$ for $k \in \mathbb{N}$ are choice functions. Clearly, by (1), $x \preccurlyeq y_{\beta}^{k}$ for every $\beta<\alpha$ and $k \in \mathbb{N}$ so that the set occurring in (2) is nonempty. So the sequences $\left\{x_{\beta}^{k}\right\}_{\beta<\alpha}$ are well defined.

These sequences are increasing. To show this consider the propositional formula $A(\beta)$ for $\beta<\alpha$ meaning: if $\gamma<\xi \leq \beta$ then $x_{\gamma}^{k} \prec x_{\xi}^{k}$ for $k \in \mathbb{N}$.

${ }^{5}$ Georg Cantor: I think of a set as a precipice. On the other hand, Leopold Kronecker brief: Cantor is the corruptor of youth. Richard Dedekind: "I think of a set as a closed sack which contains certain specified objects which one doesn't see". David Hilbert in 1925: "No one should ever drive us from the paradise which Cantor created for us". Bertrand Russel: "Thus mathematics may defined as the subject in which we never know that we are talking about, nor whether what we are saying is true". 
If $A(\gamma)$ for $\gamma<\beta$, then $\left\{x_{\delta}^{k}\right\}_{\delta<\beta}$ are chains, and consequently $x_{\delta}^{k} \leq y_{\beta}^{k}$ for $\delta<\beta$ and $k \in \mathbb{N}$. Hence, also $x_{\delta}^{k}<x_{\beta}^{k}$ for $\delta<\beta$ and $k \in \mathbb{N}$, i.e., $A(\beta)$ holds. By Principle of Transfinite Induction we have $A(\beta)$ for all $\beta<\alpha$. Now put

$$
M=\bigcup_{\beta<\alpha}\left\{x_{\beta}^{k}\right\} \quad \text { for } k \in \mathbb{N},
$$

then we have $M \subset P$, whence $\operatorname{Card}(M) \leq \operatorname{Card}(P)=m$, whereas $\operatorname{Card}(M)>$ $m$. This shows that for some $\beta<\alpha$ we must have $\left\{z \in P: y_{\beta}^{k} \prec z\right\}=\varnothing$, i.e., $y_{\beta}^{k}(k \in \mathbb{N})$ are maximal elements in $P$, and clearly $x \preccurlyeq y_{\beta}^{k}($ for $k \in \mathbb{N}$ ). The proof is complete.

Theorem 2.2. Let $P$ be a partially ordered set, then there exist at least countable functions $f_{k}$ (for $k \in \mathbb{N}$ ) or finite functions $f_{k}$ (for $k=1, \ldots, m$ and a fixed number $m \in \mathbb{N}$ ) such that for each nonempty subset $A$ of $P$ is $f_{k}(A) \in A$ for $k \in \mathbb{N}$ or $f_{k}(A) \in A$ for $k=1, \ldots, m$ and a fixed $m \in \mathbb{N}$.

Proof. (Application of the Lemma of Infinite Maximality). Let $\mathcal{A}$ be any collection of nonempty sets, and put $M=\cup \mathcal{A}$. Let $P$ be the family of those sets $F \subset M$ for which the intersection $F \cap A$ contains at most one point for every $A \in \mathcal{A}$. The set $P$ is an ordered set with inclusion $\subset$ and $\varnothing \in P$. If $\mathcal{L} \subset P$ is a chain, then $\cup \mathcal{L} \in P$. In fact, if $\cup \mathcal{L} \cap A$ for an $A \in \mathcal{A}$ contains two different elements, say $x$ and $y$, then there exist sets $D_{x}, D_{y} \in \mathcal{L}$ such that $x \in D_{x} \cap A$ and $y \in D_{y} \cap A$. But since $\mathcal{L}$ is a chain, one of the sets $D_{x}$, $D_{y}$ is contained in the other say $D_{x} \subset D_{y}$. But then $x, y \in D_{y}$ and $D_{y} \cap A$ contains more than one point.

By Theorem 2.1 there exist in $P$ maximal elements $R_{k}$ (for $k \in \mathbb{N}$ ). We will show that $R_{k} \cap A \neq \varnothing$ for every $A \in \mathcal{A}$ and $k \in \mathbb{N}$. If we had $R_{k} \cap A_{0}$ for an $A_{0} \in \mathcal{A}$ and $k \in \mathbb{N}$, then for $x_{0} \in A_{0}$ we might define a set $\mathcal{R}^{*}=R_{k} \cup\left\{x_{0}\right\}$ for $k \in \mathbb{N}$. Clearly, $\mathcal{R}^{*} \in P$ and $\mathcal{R}^{*}$ is larger than $R_{k}$ (for $k \in \mathbb{N}$ ), which is impossible, since $R_{k}$ (for $k \in \mathbb{N}$ ) are maximal elements in $P$. Thus $R_{k} \cap A$ (for $k \in \mathbb{N}$ ) is a singleton for every $A \in \mathcal{A}$ and $k \in \mathbb{N}$, and we can define functions $f_{k}: \mathcal{A} \rightarrow M$ (for $k \in \mathbb{N}$ ) by $f_{k}(A)=A \cap R_{k}$ for $k \in \mathbb{N}$. The functions $f_{k}$ (for $k \in \mathbb{N}$ ) are choice functions.

In the second case, by Theorem 2.1, there exist in $P$ a finite number maximal elements $R_{0}, R_{1}, \ldots, R_{m}$ (for a fixed $m \in \mathbb{N}$ ). We can define functions $f_{k}: \mathcal{A} \rightarrow M$ (for $k=1, \ldots, m$ ) by $f_{k}(A)=A \cap R_{k}$ for $k=1, \ldots, m$. Then the functions $f_{k}$ (for $k=1, \ldots, m$ ) are chioice functions. The proof is complete.

\section{Geometry of the Axiom of Infinite Choice}

The first specific reference to the Axiom of Choice was given in a paper by G. Peano in 1890. In 1892 R. Bettazzi published an article on discontinuous real functions with terminology of infinite many arbitrary choices. 
In this sense, we give a demonstration that the equation (Eq) has at least countable solutions or has at least finite solutions. For this see the following Figures:
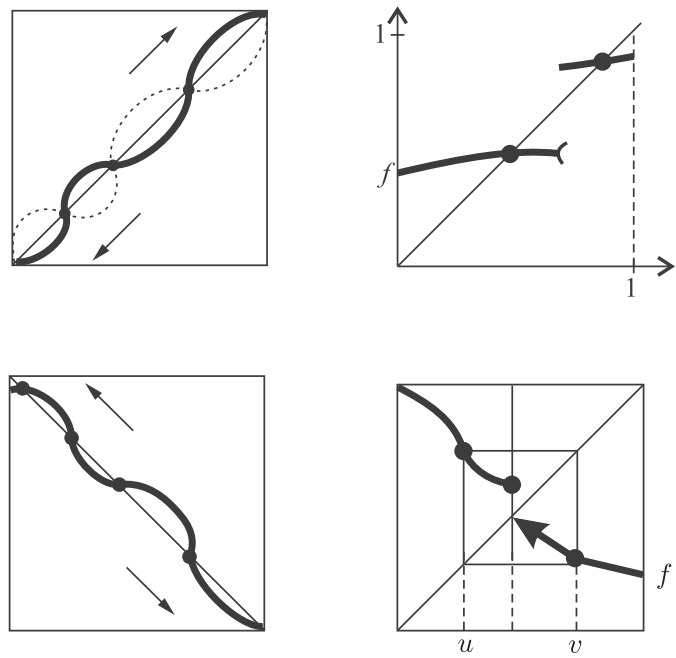

$$
u=f(v), v=f(u)
$$

also, as an example, the equation $\sin x=0$ or the equation, in an equivalent form, $x+\sin x=x$. As two statements on the equation (Eq) we obtain the following two results.

Zorn's lemma is an immediate consequence of the Lemma of Infinite Maximality, too.

As two direct consequences of the Axiom of Infinite Choice we have the following two essential results in sets theory:

1) Every isotone map of complete lattice into itself has at least countable or finite fixed points, and

2) Every antitone map of complete lattice into itself has at least countable or finite fixed apices!

Theorem 3.1. (Axiom of Infinite Choice for Points). Let $P$ be a set partially ordered by an ordering relation $\preccurlyeq$. Then the following statements are equivalent:

(a) (Lemma of Infinite Maximality). Let $P$ be an inductive partially ordered set. Then $P$ has at least countable or finite maximal elements.

(b) Let $P$ be an inductive partially ordered set and $f$ a mapping from $P$ into $P$ such that

$$
x \preccurlyeq \sup \{x, f(x)\} \text { for all } x \in \operatorname{Sub} f(P),
$$

then the function $\varphi(x):=\sup \{x, f(x)\}$ has at least countable or finite fixed points. If for all $a, b \in P$ the following condition holds in the form as

$$
\sup \{a, b\}=a \quad \text { implies } \quad a=b,
$$


then the mapping $f$ has at least countable or finite fixed points. (Also, the dually statement of this statement holds).

(c) Let $P$ be an inductive partially ordered set and $f$ a mapping from $P$ into $P$ such that

$$
x \preccurlyeq f(x) \text { for all } x \in \operatorname{Sub} f(P),
$$

then $f$ has at least countable or finite fixed points. (Also, the dually statement of this statement holds).

Theorem 3.2. (Axiom of Infinite Choice for Apices). Let $P$ be a set partially ordered by an ordering relation $\preccurlyeq$. Then the following statements are equivalent:

(a) (Lemma of Infinite Maximality). ${ }^{6}$ Let $P$ be an inductive partially ordered set. Then $P$ has at least countable or finite maximal elements.

(b) Let $P$ be an inductive partially ordered set and $f$ a mapping from $P$ into $P$ such that

$$
x \preccurlyeq \sup \left\{x, f^{2}(x)\right\} \quad \text { for all } \quad x \in \operatorname{Sub} f(P),
$$

then the function $\psi(x):=\sup \left\{x, f^{2}(x)\right\}$ has at least countable or finite fixed points. If condition (A) holds, then the mapping $f$ has at least countable or finite fixed apices. (Also, the dually statement of this statement holds).

(c) Let $P$ be an inductive partially ordered set and $f$ a mapping from $P$ into $P$ such that

$$
x \preccurlyeq f^{2}(x) \text { for all } x \in \operatorname{Sub} f(P),
$$

then $f$ has at least countable or finite fixed apices. (Also, the dually statement of this statement holds).

The proofs of these statements are very similar with the preceding proofs. Thus the proofs of this statements we omit. Also and for the next statement!

Essential Facts. We notice that Fredholm and Leray-Schauder alternatives are direct consequences of the Axiom of Infinite Choice. Also, Schauder and Brouwer theorem of fixed point (as well as Fixed Point Theory) are direct consequences of the Axiom of Infinite Choice.

In connection with the Axiom of Infinite Choice I have the following direct result as an extension of the Schauder problem (Theorem 7.1).

\footnotetext{
${ }^{6}$ What were the beginnings of Zorn's principle? According to his later reminiscences, he first formulated it at Hamburg in 1933, where Claude Chevalley and Emil Artin then took it up as well. Indeed, when $\mathrm{Zorn}$ applied it to obtain representatives from certain equivalence classes on a group, Art inr ecognized that Zorn's principle yields the Axiom of Choice. By late in 1934, Zorn's principle had found users in the United States who dubbed in Zorn's lemma. In October, when Zorn lectured on his principle to the American Mathematical Society in New York, Solomon Lefschetz recomended that Zorn publish his result. The paper appeared, the following year, in 1935.
} 
Theorem 3.3. Let $C$ be a nonempty convex compact subset of a linear topological space $X$ and suppose $T: C \rightarrow C$ is a continuous mapping. Then $T$ has at least countable fixed points or $T$ has at least finite fixed points.

Open problem. We notice that in 1963 P. J. Cohen proved that the Axiom of Choice is independent of the remaining Axioms of the Sets Theory. In this sense is the Axiom of Infinite Choice is independent of the remaining Axioms of the Sets Theory? (I think that the answer is yes!?)

Also, in connection with this, I have the following direct result as an extension of the Recursion Theorem.

Theorem 3.4. If $\xi$ is an element of a set $X$, and if $f$ is a function from $X$ into $X$, then there exist at least countable functions $f_{k}($ for $k \in \mathbb{N})$ or there exist at least finite functions $f_{k}($ for $k=1, \ldots, m$ and a fixed number $m \in \mathbb{N})$ from $\mathbb{N}$ into $X$ such that $f_{k}(0)=\xi$ and such that $f_{k}(n \cup\{n\})=f\left(f_{k}(n)\right)$ for all $n \in \mathbb{N}$.

\section{Forked Points on Topological Spaces}

Let $X$ be an arbitrary nonempty set, $T$ be a mapping from $X$ into $X$, and $P:=(P, \preccurlyeq)$ a nonempty partially ordered set. A mapping $f: X \rightarrow P$ (or $f: X \rightarrow X$ ) has a forked point (or furcate point) $p \in X$ if the following equality holds in the form

$$
f(p)=f(T p) \text { for some } p \in X ;
$$

frequently, we say that in this case (Ra), the mapping $f: X \rightarrow P$ has a pair $(p, T p)$ of bifurcation points, or that $T: X \rightarrow X$ has a forks point $p \in X$.

We notice that many problems in nonlinear functional analysis (as and in the fixed point theory) are reducible to the existence of forked points of certain mappings.

Further, let $P:=(P, \preccurlyeq)$ be a partially ordered set with a minimum (or with the property that every nonempty subset in $P$ has an infimum) such that every decreasing sequence $\left\{x_{n}\right\}_{n \in \mathbb{N}}$ in $P$ has a limit in $P$, denoted by $\lim _{n \rightarrow \infty} x_{n}$.

In connection with this, we shall introduce the concept of lower ordered RBS-convergence in a topological space $X$ for $B: X \rightarrow P$, i.e., a topological space $X$ satisfies the condition of lower ordered RBS-convergence iff $\left\{a_{n}(x)\right\}_{n \in \mathbb{N}}$ is an arbitrary sequence in $X$ with arbitrary $x \in X$ and if $B\left(a_{n}(x)\right) \rightarrow b=b(x) \in P(n \rightarrow \infty)$ implies that $\left\{a_{n}(x)\right\}_{n \in \mathbb{N}}$ has a convergent subsequence $\left\{a_{n(k)}(x)\right\}_{k \in \mathbb{N}}$ which converges to $\xi \in X$, where

$$
B(\xi) \preccurlyeq \inf _{x \in X} \lim _{k \rightarrow \infty} B\left(a_{n(k)}(x)\right) .
$$

In this part of the paper, we apply the technics of maximal elements to the equations of the forks theory. As an immediate consequence of the Lemma of Infinite Maximality we obtain the following ordered principle. 
In this sense, let $X$ be a topological space, Define a relation $\preccurlyeq_{o r k}$ on $X$ by the following conditions:

$$
\begin{cases}x \preccurlyeq{ }_{\text {ork }} y & \text { if and only if } \quad B(x) \preccurlyeq B(y), \\ x=_{\text {ork }} y & \text { if and only if } \quad B(x)=B(y),\end{cases}
$$

where $B: X \rightarrow P$ is a function with the given conditions.

It is verify that $\preccurlyeq_{o r k}$ is a partial ordering (asymmetric and transitive relation) in $X$. The poset $X$ together with this partial ordering, is denoted by $X_{\text {ork }}$.

Theorem 4.1. (Ordering Principle). Let $X$ be a topological space with the poset $X_{\text {ork }}$. If $X$ satisfies the condition of lower ordered $R B S$-convergence, then $X_{\text {ork }}$ has at least countable or finite minimal elements $z_{k} \in X_{\text {ork }}$ with $z_{k} \preccurlyeq$ ork $x$ for given $x \in X_{\text {ork }}$.

Sketck proof. Let $C$ be a chain in $X_{\text {ork }}$ and now let $t \in C$ be given. Denote by $\alpha:=\inf \{B(x): x \in C\}$. Then the set $M(x, n)$ of all $y \in C$ with $x \preccurlyeq$ ork $y$ and $\alpha \prec B(y) \prec \alpha_{n}\left(\alpha_{n} \rightarrow \alpha\right)$ is nonempty for each $n \in \mathbb{N}$ and $x \in C$. Let $I$ be a choice function for the family of all nonempty subsets of $C$. Then, by the recursion theorem, there is a sequence $\left\{x_{n}\right\}_{n \in \mathbb{N}}$ in $C$ such that $x_{0}=t$ and $x_{n+1}=I\left(M\left(x_{n}, n\right)\right)$ for $n \in \mathbb{N}$. This implies (from lower ordered RBS-convergence) that there exists $\xi \in X_{\text {ork }}$ such that $B(\xi) \preccurlyeq \cdots \preccurlyeq B\left(x_{n}\right)$ for $n \in \mathbb{N}$. Now let $x \in C$. Then we can find an $i \in \mathbb{N}$ such that $B(\xi) \preccurlyeq B\left(x_{i}\right) \prec \alpha_{i} \preccurlyeq B(x)$. Since $x$ and $x_{i}$ are in the chain $C$, we obtain $\xi \preccurlyeq$ ork $x$. This shows that $\xi$ is a minorant of $C$. By the nature of $C$ (by Lemma of Infinite Maximality) it follows that there is at least countable or finite $z_{k} \in X_{\text {ork }}$ which are minimal in $X_{\text {ork }}$.

We notice that the proof of this statement is totally an analogy with the former proofs of ordered principles.

As an immediate consequence of Theorem 4.1 (Ordering Principle) we obtain the following result in the forks theory.

Theorem 4.2. (Forked points existence, Tasković [2005]). Let $T$ be a mapping of a topological space $X$ into itself, where $X$ satisfies the condition of lower ordered $R B S$-convergence. If

$$
B(T x) \preccurlyeq B(x) \quad \text { for every } \quad x \in X,
$$

then for $T$ there exist at least countable or finite forked points $\xi_{k} \in X$, i.e., then the following equalities hold in the form

$$
B\left(T \xi_{k}\right)=B\left(\xi_{k}\right)=\alpha_{k}:=\inf _{x \in X} \lim _{n \rightarrow \infty} B\left(b_{n}^{k}(x)\right)
$$

for some sequences $\left\{b_{n}^{k}(x)\right\}_{n \in \mathbb{N}}$ in $X$ which converges to the forked points $\xi_{k} \in X$.

A brief proof of a variant of this statement based on some elementary facts may be found in Tasković [2005]. For this, also see Tasković [2001]. 
Proof of Theorem 4.2. Consider the partially ordered set $X_{\text {ork }}$ and let $\xi_{k}$ be minimal elements. Using $(\mathrm{Bu})$, it also following from (fk) that $T x \preccurlyeq_{o r k} x$ for all $x \in X$ and $T \xi_{k} \preccurlyeq_{o r k} \xi_{k}$ in $X_{\text {ork }}$ and, because $\xi_{k}$ are minimal it follows that $T \xi_{k}={ }_{o r k} \xi_{k}$. The proof is complete.

In this sense, we shall introduce the concept of lower BCS-convergence in a topological space $X$ for $B: X \rightarrow \mathbb{R}_{+}^{0}$, i.e., a topological space $X$ satisfies the condition of lower BCS-convergence (orbital lower BCS-convergence) if $\left\{a_{n}(x)\right\}_{n \in \mathbb{N}}$ is an arbitrary sequence (an arbitrary iteration sequence) in $X$ with arbitrary $x \in X$ and if $B\left(a_{n}(x)\right) \rightarrow b=b(x) \geqslant 0(n \rightarrow \infty)$ implies that $\left\{a_{n}(x)\right\}_{n \in \mathbb{N}}$ has a convergent subsequence $\left\{a_{n(k)}(x)\right\}_{k \in \mathbb{N}}$ which converges to $\xi \in X$, where

$$
B(\xi) \leqslant \inf _{x \in X} \liminf _{k \rightarrow \infty} B\left(a_{n(k)}(x)\right) .
$$

Theorem 4.3. (Monotone Principle of Forked Points). Let $T$ be a mapping of a topological space $X$ into itself, where $X$ satisfies the condition of orbital lower BCS-convergence. If

$$
B(T x) \leq B(x) \quad \text { for every } \quad x \in X,
$$

then for $T$ there exist at least countable or finite forked points $\xi_{t} \in X$, i.e., then the following equalities hold in the form

$$
B\left(T \xi_{t}\right)=B\left(\xi_{t}\right)=\alpha_{t}:=\inf _{x \in X} \lim _{n \rightarrow \infty} B\left(b_{n}^{t}(x)\right)
$$

for some sequence $\left\{b_{n}^{t}(x)\right\}_{n \in \mathbb{N}}$ in $X$ which converges to $\xi_{t}$. In this case, the point $\xi_{t} \in X$ is a minimal element of the set $X_{B, i d}$ with the property $\xi_{t}={ }_{B, i d} T \xi_{t}$.

Annotations. A fine illustration for Theorem 4.3 is a well known statement in 1936 which was given by Freudenthal and Hurewicz in the following form: If $(X, \rho)$ is a compact metric space and if $T$ is a mapping of $X$ into itself such that

$$
\rho[T(x), T(y)]<\rho[x, y] \quad \text { for all } \quad x, y \in X \quad(x \neq y),
$$

then the mapping $T$ has a unique fixed point $\xi \in X$.

Indeed, first, since $X$ is a compact space it follows that the condition of lower (orbital) BCSconvergence holds. Second, let $B(x):=\rho(x, T x)$, thus applying Theorem 4.3 we have that there exists $\xi \in X$ such that (M). But, from (3) for $\xi \neq T \xi$ we obtain

$$
B(\xi)=B(T \xi)=\rho\left[T(\xi), T^{2}(\xi)\right]<\rho[\xi, T(\xi)]=B(\xi),
$$

i.e., we obtain a contradiction. This means that $\xi=T \xi$ for some $\xi \in X$. The uniqueness follows immediately from (3). The proof is complete.

In connection with this statement of Freudenthal-Hurewicz [1936] there exist more extensions. An extension of this statement to give Edelstein [1962] to change the compactness with the following weak condition in the form: if $\left\{T^{n}(x)\right\}_{n \in \mathbb{N}}$ is an arbitrary iteration sequence in $X$ with arbitrary $x \in X$, then he has at least one convergent subsequence in $X$.

On the other hand we notice that this result of Freudenthal and Hurewicz in 1936 appeared, also, at the same year independently by Niemytzki [1936].

These facts are direct examples for the preceding Theorem 4.3. Also, this facts can be great for further considerations and in the fixed point theory. 
Annotation. We notice that in Theorem 4.3 the condition (B) we can change with a sectional condition in the following sense, that there exists $z \in X$ such that

$$
B(T x) \leq B(x) \text { for every } \quad x \in O(z),
$$

where $O(z)$ is an orbit of the point $z \in X$ for the mapping $T$ of a topological space $X$ into itself. The proofs in this case are almost one and the same thing.

As an immediate consequence of Theorem 4.3 we obtain the following direct generalization of Caristi's fixed point theorem (=Theorem 4.4 in the case $k=0)$.

Theorem 4.4. Let $T$ be a self-map on a complete metric space $(X, \rho)$. Suppose that there exists a lower semicontinuous function $G: X \rightarrow \mathbb{R}_{+}^{0}$ and an arbitrary fixed integer $k \geqslant 0$ such that

$$
\rho[x, T x] \leqslant G(x)-G(T x)+\cdots+G\left(T^{2 k} x\right)-G\left(T^{2 k+1} x\right)
$$

and $G\left(T^{2 i+1} x\right) \leq G\left(T^{2 i} x\right)$ for $i=0,1, \ldots, k$ and for every $x \in X$. Then $T$ has at least countable or finite fixed points $\xi_{t}$ in $X$.

As an immediate consequence of the preceding result of Theorem 4.4 we obtain the following statement of a fixed point.

Theorem 4.5. Let $T$ be a self-map on a complete metric space $(X, \rho)$. Suppose that there exists a lower semicontinuous function $G: X \rightarrow \mathbb{R}_{+}^{0}$ such that

$$
\rho[x, T x] \leqslant \sum_{i=0}^{+\infty}\left(G\left(T^{2 i} x\right)-G\left(T^{2 i+1} x\right)\right)
$$

and $G\left(T^{2 i+1} x\right) \leq G\left(T^{2 i} x\right)$ for $i \in \mathbb{N} \cup\{0\}$ and for every $x \in X$. Then $T$ has at least countable or finite fixed points $\xi_{t}$ in $X$.

Further interpretations on the forks theorems. We notice, in this section, that for the preceding main statements we can give their following explanations via the BCS-completeness and the lower BCS-continuous in the following sense.

In connection with this, we shall introduce the concept of BCS-completeness in a space $X$ for a function $B: X \rightarrow \mathbb{R}$, i.e., a topological space $X$ is called BCS-complete (orbital BCS-complete) iff $\left\{a_{n}(x)\right\}_{n \in \mathbb{N}}$ is an arbitrary sequence (an arbitrary iteration sequence) in $X$ with arbitrary $x \in X$ and if $B\left(a_{n}(x)\right) \rightarrow b=b(x) \in \mathbb{R} \cup\{ \pm \infty\}$ as $n \rightarrow \infty$ implies that $\left\{a_{n}(x)\right\}_{n \in \mathbb{N}}$ has a convergent subsequence in $X$.

On the other hand, a function $B: X \rightarrow \mathbb{R}$ is lower BCS-continuous (orbital lower BCScontinuous) at $p \in X$ iff $\left\{a_{n}(x)\right\}_{n \in \mathbb{N}}$ is an arbitrary sequence (an arbitrary iteration sequence) in $X$ with arbitrary $x \in X$ and if $a_{n}(x) \rightarrow p(n \rightarrow \infty)$ implies that is

$$
B(p) \leqslant \inf _{x \in X} \liminf _{n \rightarrow \infty} B\left(a_{n}(x)\right) .
$$

We are now in a position to formulate the following explanations of the preceding theorems as corresponding equivalent forms:

Theorem 4.3a. Let $T$ be a mapping of a topological space $X$ into itself and let $X$ be orbital BCS-complete. If (B) holds and if $B: X \rightarrow \mathbb{R}_{+}^{0}$ is an orbital lower BCS-continuous functional, then there exist at least countable or finite furcate points $\xi_{t} \in X$.

The proof of this statement is totally analogous with the proof of Theorem 4.3 which is equivalent, in the booking, to the Theorem $4.3 \mathrm{a}$. 
Open problem. Let $T$ be a mapping of a topological space $X$ into itself, where $X$ satisfies the condition of orbital lower BCS-convergence. If it is supposed that there exist a lower semicontinuous function $G: X \rightarrow \mathbb{R}_{+}^{0}$, a function $A_{p}: X \times X \rightarrow \mathbb{R}_{+}^{0}$ (for $p \in \mathbb{R}$ ), and an arbitrary fixed integer $k \geqslant 0$ such that

$$
A_{p}(x, T x) \leq G(x)-G(T x)+\cdots+G\left(T^{2 k} x\right)-G\left(T^{2 k+1} x\right)
$$

and $G\left(T^{2 i+1} x\right) \leq G\left(T^{2 i} x\right)$ for $i=0,1, \ldots, k$ and for every $x \in X$, does $T$ have at least one fixed point in the topological space $X$ ?

In connection with this problem, we notice that in a special case of this open problem, if $X:=(X, \rho)$ is a complete metric space, if $A_{p}(x, T x)=(\rho[x, T x])^{p}$, and if $k=1$ in (E), i.e., if

$$
(\rho[x, T x])^{p} \leq G(x)-G(T x) \text { for every } \quad x \in X
$$

and $p \in \mathbb{R}$, then the open problem of $\mathrm{Kirk}[1976]$ is settled in the negative if $0<p<1$, and positive if $p \geqslant 1$ (see: Bae-Park [1983]). Another counter example (for $p<1$ ) is given in Khamsi-Misane [1995].

Open problem. We notice that the preceding proof of Theorem 4.5 is given without direct application of some of maximal principles as well as without Zorn's lemma and Lemma of Infinite Maximality. Can a new proof of Theorem 4.5 be given elementary without Axiom of Infinite Choice?

Further, we notice that the preceding monotone principle of forked point appears before the monotone principle of fixed point which in 1985 for the first time appeared from Tasković [1985]. For the next appearence of this monotone principle see Tasković [1990].

In this sense, let $X$ be a topological space, $T: X \rightarrow X$, and let $A$ : $X \times X \rightarrow \mathbb{R}_{+}^{0}:=[0,+\infty)$. A topological space $X$ satisfies the condition of TCS-convergence iff $x \in X$ and if $A\left(T^{n} x, T^{n+1} x\right) \rightarrow 0(n \rightarrow \infty)$ implies that the iterates sequence $\left\{T^{n} x\right\}_{n \in \mathbb{N}}$ has a convergent subsequence in $X$.

For $x \in X, \sigma(x, \infty):=\left\{x, T x, T^{2} x, \ldots\right\}$ is called the orbit of $X$. A function $f$ mapping $X$ into the reals is $f$-orbital lower semicontinuous at $p \in X$ if $\left\{x_{n}\right\}_{n \in \mathbb{N}}$ is a sequence in $\sigma(x, \infty)$ and $x_{n} \rightarrow p(n \rightarrow \infty)$ implies that $f(p) \leqslant \liminf _{n \rightarrow \infty} f\left(x_{n}\right)$. The following fact holds.

Lemma 4.1. (Tasković, [1978]). Let the mapping $\varphi: \mathbb{R}_{+}^{0} \rightarrow \mathbb{R}_{+}^{0}$ have the following properties

$$
\left(\forall t \in \mathbb{R}_{+}:=(0,+\infty)\right)\left(\varphi(t)<t \quad \text { and } \quad \limsup _{z \rightarrow t+0} \varphi(z)<t\right) .
$$

If the sequence $\left(x_{n}\right)$ of real numbers satisfies the condition $x_{n+1} \leq \varphi\left(x_{n}\right)$ for every $n \in \mathbb{N}$, then it converges to zero.

Theorem 4.6. (Monotone Principle of Fixed Point). ${ }^{7}$ Let $T$ be a mapping of a topological space $X$ into itself, where $X$ satisfies the condition of TCSconvergence. Suppose that there exists a mapping $\varphi: \mathbb{R}_{+}^{0} \rightarrow \mathbb{R}_{+}^{0}$ such that $(I \varphi)$ and

$$
A(T x, T y) \leqslant \varphi(A(x, y)) \quad \text { for all } \quad x, y \in X
$$

${ }^{7}$ We notice that DjuroKurepa in 1971, first version of my Monotone Principle of Fixed Point, has been sent to Professor JeanLeray (Paris) for the opinion. Some of Leray's ideas I am to realize in several published papers. In general form for the first time, fundamental elements of Monotone Principle I give in: Proc. Amer. Math. Soc., 94 (1985), 427-432. For later facts on this see: Tasković [1990]. 
where $A: X \times X \rightarrow \mathbb{R}_{+}^{0}, x \mapsto A(x, T x)$ is T-orbital lower semicontinuous or $T$ is orbital continuous, and $A(a, b)=0$ implies $a=b$. Then $T$ has a unique fixed point $\xi \in X$ and $T^{n} x \rightarrow \xi$ for each $x \in X$.

Proof. (Application of Theorem 4.3). Let $B(x):=A(x, T x)$ which is lower semicontinuous on $X$, then $B(T x) \leq B(x)$ for every $x \in X$, i.e., (B) in Theorem 4.3. Since $X$ satisfies the condition of orbital lower BCS-convergence (because from Lemma 4.1 it follows that $A\left(T^{n} x, T^{n+1} x\right) \rightarrow 0$ as $n \rightarrow \infty$ and thus, by TCS-convergence, $\left\{T^{n}(x)\right\}_{n \in \mathbb{N}}$ has a convergent subsequence $\left\{T^{n(k)}(x)\right\}_{k \in \mathbb{N}}$ to $\xi \in X$ and $(\mathrm{Bi})$ holds by lower semicontinuous of $B$ ), applying Theorem 4.3 we obtain $B(T \xi)=B(\xi)=A(\xi, T \xi):=\alpha$. Thus from (MP) for $\alpha \neq 0$ we have

$$
\alpha=A\left(T \xi, T^{2} \xi\right) \leqslant \varphi(A(\xi, T \xi))<A(\xi, T \xi)=\alpha
$$

for some $\xi \in X$. This is a contradiction, thus $\alpha=0$, i.e., $\xi=T \xi$ for some $\xi \in X$. The uniqueness follows immediately from (MP). The proof is complete.

Further, let $X$ be a topological space, let $T: X \rightarrow X$, and let $B$ : $X \rightarrow \mathbb{R}_{+}^{0}$ be a given function. In connection with the preceding, in 1985 we investigated the concept of local TCS-convergence in a space $X$, i.e., a topological space $X$ satisfies the condition of local TCS-convergence iff $x \in X$ and if $B\left(T^{n} x\right) \rightarrow 0(n \rightarrow \infty)$ implies that the iterates sequence $\left\{T^{n} x\right\}_{n \in \mathbb{N}}$ has a convergent subsequence in $X$.

We notice that the condition of TCS-convergence is to set task for the function $(x, y) \mapsto A(x, y)$, till the condition of local TCS-convergence is to set task for the function $x \mapsto B(x)$.

Facts on TCS-convergence. For the first time in 1985 I introduced the conditions of TCSconvergence and local TCS-convergence with the intention to transmit it to the properties of Cauchy sequence from metric spaces on topological spaces, see Tasković [1985].

We can briefly say, in connection with this, that the results of forked points are based on RBS-convergence and BCS-convergence. It is a new viewpoint which is an extension of the TCSconvergence.

At the interval of the next seven years more authors have considered appearance of TCSconvergence as a special case od the property TCS-convergence, precisely, in this way, $d$-completeness of topological spaces, see: Hicks [1992], Hicks-Rhoades [1992], Saliga [1996], and Popa [2002].

Recently, 10 years next appeared Monotone Principle in 1985, in connection with this $\mathrm{J}$ a chy mski, Matkowski, and Swiatkowski [Journal of Applied Analysis, 1 (1995), 125-134, Theorem 1, p. 130] proved a very special case of Monotone Principle of Fixed Point on Hausdorff spaces. For the same also see: A a mri-Moutawakil [2003].

As a further application of Theorem 4.3 (Monotone principle of forked points) we obtain the following well-known result in the fixed point theory by Tasković [1985]. 
Theorem 4.7. (Localization Monotone Principle). ${ }^{8}$ Let $T$ be a mapping of a topological space $X$ into itself, where $X$ satisfies the condition of local TCS-convergence. Suppose that there exists a mapping $\varphi: \mathbb{R}_{+}^{0} \rightarrow \mathbb{R}_{+}^{0}$ such that $(I \varphi)$ and

$$
B(T x) \leqslant \varphi(B(x)) \quad \text { for every } \quad x \in X,
$$

where $B: X \rightarrow \mathbb{R}_{+}^{0}$ is $T$-orbital lower semicontinuous or $T$ is orbital continuous, and $B(x)=0$ implies $T x=x$. Then $T$ has at least countable or finite fixed points $\xi_{t} \in X$.

A brief proof of this statement based on Ordering Principle (Theorem 4.1) may be found in Tasković [1988]. The first proof which is elementary may be found in Tasković [1985]. We notice that this result was generalized by Turinici [1993] as a further localization. The following proof is an application of Monotone Principle of Forked Point.

The localization of monotone principle of a fixed point is a common generalization of results of: Banach [1922], Browder [1968], Boyd-Wong [1969], Dugundji-Granas [1978], Krasnoselskij et al. [1973], Caristi [1976], Kirk [1976], Matkowski [1975], Hicks-Rhoades [1979], Fisher [1976], and many others.

Recently, 15 years later appeared Localization Monotone Principle in 1985, in connection with this Suzuki [J. Math. Anal. Appl. 253 (2001), 440-458, Theorem 1, p. 451] which proved a very special case of Localization Monotone Principle of Fixed Point.

Proof of Theorem 4.7. (Application of Theorem 4.3). Let $x$ be an arbitrary point in $X$. The function $B: X \rightarrow \mathbb{R}_{+}^{0}$ is lower semicontinuous and satisfies, by $(\mathrm{I} \varphi), B(T x) \leq B(x)$ for every $x \in X$, i.e., the inequality (B) in former Theorem 4.3.

Applying Lemma 4.1 to the sequence $\left\{B\left(T^{n} x\right)\right\}_{n \in \mathbb{N}}$ we obtain that $B\left(T^{n} x\right) \rightarrow$ $0(n \rightarrow \infty)$, and thus $X$ satisfies the condition of orbital lower BCSconvergence with the inequality $(\mathrm{Bi})$. Applying Theorem 4.3 we have $B\left(T \xi_{t}\right)=$ $B\left(\xi_{t}\right):=\alpha_{t}$ for some $\xi_{t} \in X$. Also, from $(\mathrm{I} \varphi)$, for $\alpha_{t} \neq 0$ we have

$$
\alpha_{t}=B\left(\xi_{t}\right)=B\left(T \xi_{t}\right) \leqslant \varphi\left(B\left(\xi_{t}\right)\right)<B\left(\xi_{t}\right)=\alpha_{t}
$$

for some $\xi_{t} \in X$. This is a contradiction, which means that $\alpha_{t}=0$, i.e., $\xi_{t}=T \xi_{t}$ for some $\xi_{t} \in X$. The proof is complete.

An important event. We notice that the preceding facts and consequences are an affirmation that Monotone Principle of Forked Points is a natural extension of the Monotone Principle of Fixed Point, and Localization Monotone Principle of Fixed Points.

Some new geometric theorems. As further applications of the preceding Theorem 4.3, on forked points, we obtain the following geometric statements of fixed points on complete metric spaces.

\footnotetext{
${ }^{8}$ For monotone principles, specially for Localization Monotone Principle of Fixed Point, James Dugundji, in the letter for me of October 5 in 1984, briefly among the rest writes, that he is convinced of the role of Localization Monotone Principle in the fixed point theory (and nonlinear functional analysis). This opinion of J. Dugundji has been confirmed many a time, via various phenomena, as one can see from many results proven in this book.
} 
Theorem 4.8. Let $T$ be a self-map on a complete metric space $(X, \rho)$. Suppose that there exists a lower semicontinuous function $G: X \rightarrow[a,+\infty)$ for some $a>0$ such that

$$
\rho[x, T x] \leqslant \frac{1}{G(T x)}-\frac{1}{G(x)} \quad \text { for every } \quad x \in X,
$$

then $T$ has at least countable or finite fixed points $\xi_{t}$ in $X$. (This result is an analogy with Caristi's theorem).

The proof of this statement (without utilizing Axiom of Infinite Choice) is analogous to the proof of based on Theorem 4.3.

As a direct extension of Theorem 4.8, from Theorem 4.3, we obtain the following result of a fixed point on complete metric spaces.

Theorem 4.9. Let $T$ be a self-map on a complete metric space $(X, \rho)$. Suppose that there exists a lower semicontinuous function $G: X \rightarrow[a,+\infty)$ for some $a>0$ and an arbitrary fixed integer $k \geqslant 0$ such that

$$
\rho[x, T x] \leqslant \frac{1}{G(T x)}-\frac{1}{G(x)}+\cdots+\frac{1}{G\left(T^{2 k+1} x\right)}-\frac{1}{G\left(T^{2 k} x\right)}
$$

and $G\left(T^{2 i+1} x\right) \leq G\left(T^{2 i} x\right)$ for $i=0,1, \ldots, k$ and for every $x \in X$. Then $T$ has at least countable or finite fixed points $\xi_{t}$ in $X$.

We notice that for $k=0$ in Theorem 4.9 we have Theorem 4.8. Proof of this statement is based on Theorem 4.2.

In connection with the preceding, as a direct consequence of Theorem 4.9, we obtain the following statement of fixed point on complete metric spaces.

Theorem 4.10. Let $T$ be a self-map on a complete metric space $(X, \rho)$. Suppose that there exists a lower semicontinuous function $G: X \rightarrow[a,+\infty)$ for some $a>0$ such that

$$
\rho[x, T x] \leqslant \sum_{i=0}^{+\infty}\left(\frac{1}{G\left(T^{2 i+1} x\right)}-\frac{1}{G\left(T^{2 i} x\right)}\right)
$$

and $G\left(T^{2 i+1} x\right) \leq G\left(T^{2 i} x\right)$ for $i \in \mathbb{N} \cup\{0\}$ and for every $x \in X$. Then $T$ has at least countable or finite fixed points $\xi_{t}$ in $X$.

The proof of this statement is totally analogous with the proof of Theorem 4.5. Precisely, from the Theorem 4.10, we obtain directly Theorem 4.9 as a consequence.

\section{Functions of $A$-VARiation}

Let $X$ be a nonempty set, let $T: X \rightarrow X$, and let $A: X \times X \rightarrow \mathbb{R}_{+}^{0}$ be a given function. Further, a function $T$ satisfies the condition of $A$ variation iff there exists a function $A: X \times X \rightarrow \mathbb{R}_{+}^{0}$ such that

$$
\sum_{i=0}^{\infty} A\left(T^{i}(x), T^{i+1}(x)\right)<+\infty
$$


for every $x \in X$. If $(X, \rho)$ is a metric space and $A:=\rho$, in the preceding case (4), then we say that $T$ is a bounded variation mapping or $\rho$-variation mapping.

Proposition 5.1. (Tasković in 1993). Let $X$ be a nonempty set, let $T$ : $X \rightarrow X$, and let $A: X \times X \rightarrow \mathbb{R}_{+}^{0}$ be a given mapping. Then the following facts are equivalent:

(V) $T$ is a mapping of A-variation on the nonempty set $X$ in attitude toward a given mapping $A: X \times X \rightarrow \mathbb{R}_{+}^{0}$.

(F) There exists a function $G: X \rightarrow \mathbb{R}_{+}^{0}$ such that the following inequality holds in the form that is

$$
A(x, T x) \leqslant G(x)-G(T x) \text { for all } x \in X .
$$

(S) There exists a sequence of nonnegative real functions $x \mapsto C_{n}(x, T x)$ such that the following inequality holds in the form

$$
A\left(T^{n} x, T^{n+1} x\right) \leq C_{n}(x, T x) \text { for all } x \in X,
$$

and for all $n \in \mathbb{N} \cup\{0\}$, where the series of the form $\sum_{n=0}^{\infty} C_{n}(x, T x)$ converges for all $x \in X$.

A brief proof of this statement may be found in: Tasković [1993, p. 407] and Tasković [1998]. Also see: Eisenfeld-Lakshmikantham [1977]. On the other hand, the following fact holds. Namely, if $X$ is a nonempty set, $T: X \rightarrow X$, and if $A: X \times X \rightarrow \mathbb{R}_{+}^{0}$ is a given mapping, then the following facts are equivalent: $(\mathrm{V}),(\mathrm{F}),(\mathrm{S})$ and:

(R) There exists a function $G: X \rightarrow \mathbb{R}_{+}^{0}$ and an arbitrary fixed integer $k \geqslant 0$ such that the following inequality holds in the form that is

$$
A(x, T x) \leqslant G(x)-G(T x)+\cdots+G\left(T^{2 k} x\right)-G\left(T^{2 k+1} x\right)
$$

and $G\left(T^{2 i+1} x\right) \leq G\left(T^{2 i} x\right)$ for $i=0,1, \ldots, k$ and for every $x \in X$. (The proof of this statement is totally analogous with the proof of Proposition 5.1).

We are now in a position to formulate our next consequence of Theorem 4.1 or Theorem 4.2. This statement is characteristic for the $A$-variation mappings from Tasković [1993].

Theorem 5.1. (A-variation Principle). Let $T$ be an $A$-variation mapping of topological space $X$ into itself, where $X$ satisfies the condition of TCS-convergence. If $x \mapsto A(x, T x)$ is T-orbital lower semicontinuous and $A(a, b)=0$ implies $a=b$, then $T$ has at least countable or finite fixed points.

Proof. The condition (F) implies condition (B) of Theorem 4.3. Thus, applying Theorem 4.3 we obtain this statement.

Two immediate and direct corollaries of the preceding main statement are the following two statements which is characteristic for metric spaces.

Theorem 5.2. Let $T$ be a self-map of an orbital complete metric space $(X, \rho)$. If $T$ is orbital continuous and an $\rho$-variation mapping, then $T$ has at least countable or finite fixed points. 
Theorem 5.3. Let $(X, \rho)$ be a complete metric space, and $T: X \rightarrow X$ a bounded variation mapping, i.e., an $\rho$-variation mapping. If $T$ is a continuous mapping, then $T$ has at least countable or finite fixed points.

Proof of Theorem 5.2. Since $X$ satisfies the condition of TCS-convergence ( $X$ is orbital complete metric space and $T$ is $\rho$-variation), from the preceding facts, applying Theorem 5.1 gives $T \xi_{k}=\xi_{k}$ for some $\xi_{k} \in X$.

Corollary 5.1. (Banach Contraction Principle, Banach [1922]). Let $(X, \rho)$ be a complete metric space and $T: X \rightarrow X$ is a contractive mapping. Then $T$ has a unique fixed point $\xi \in X$, and $T^{n} x \rightarrow \xi(n \rightarrow \infty)$ for each $x \in X$.

Proof. From the condition of contraction, it is easy to see that $T$ is bounded variation, i.e., $\rho$-variation. Precisely, every contraction mapping is bounded variation and continuous. Hence, it follows from the Theorem 5.1 that $T$ has a unique fixed point.

On the other hand, we notice from Theorems 5.1, 5.2 and 5.3 that our central idea is, that every continuous, bounded variation mapping of complete metric space into itself has at least countable or finite fixed points by Tasković [1993], is essential. In this sense, directly, we have the preceding Banach's contraction principle, and the following extension of Caristi's fixed point theorem.

Corollary 5.2. (Tasković, [1993]). Let T be a mapping of a topological space $X$ into itself with the property of TCS-convergence. If there is a function $G: X \rightarrow \mathbb{R}_{+}^{0}$ such that

$$
A(x, T x) \leq G(x)-G(T x) \text { for all } x \in X,
$$

where $x \mapsto A(x, T x)$ is a lower semicontinuous function or $T$ is orbital continuous, and $A(a, b)=0$ implies $a=b$, then $T$ has at least countable or finite fixed points in $X$.

In recent years a great number of papers have presented generalizations of the three fundamental famous principles of Brouwer, Schauder and Banach. In this part of the book we extend these three central results in a fixed point theory. Our central idea is, that every continuous, bounded variation mapping of complete metric space into itself has a fixed point. This fixed point theorem is a common generalization of results of Brouwer, Schauder, Banach, Sadovskij, Browder, Krasnoselskij, Darbo, Dugundji, Granas, Kirk, Caristi, Ky Fan and some others.

Brouwer's theorem $\mathbf{- 9 7 t h}$ next. Perhaps the most important of all fixed point theorems is the famous theorem of Brouwer ${ }^{9}$ in 1909 which states that every continuous mapping of the closed unit ball of the Euclidean space $\mathbb{R}^{n}$ into itself has a fixed point.

Schauder's theorem in 1927 is a generalization of Brouwer's theorem to infinite dimensional normed linear space. Schauder's theorem states that every continuous mapping of a compact convex subset of a normed linear space into itself has a fixed point.

In recent years a great number of papers have presented generalizations of the well-known essential Brouwer principle. Some of these generalizations refer to the result containing the Schauder fixed point theorem.

${ }^{9}$ Luitzen Egbertus Jan Brouwer (1881-1966) - German-Dutch mathematician, is one of the greatest mathematicians. He was a professor at the University of Amsterdam, and he is supposed to be one of the founders of modern topology. Brouwer proved the topological invariance of the dimension of the Cartesian space. Furthermore he is famous for his contributions to the Fundaments of Mathematics. But his most important result is the fixed point theorem mentioned above. 
Brouwer's theorem is one of the oldest and best known results in topology. It was proved for $n=3$ by L. Brouwer in 1909. An equivalent result was established earlier in 1904 by Bohl.

In 1920 it was Hadamard who gave (using the Kronecker index) the first proof for arbitrary $n$. Soon afterwards in 1912 Brouwer had another proof using the simplistic approximation technique and notion of the degree. Other proofs depending on various definitions of degree were also given by Alexander in 1922 and Birkhoff-Kellogg in 1922.

A simple and short proof of the Brouwer theorem based on combinatorial technique and Sperner's lemma in 1928, was given by Knaster-Kuratowski-Mazurkiewicz in 1929.

\section{Maximal Principles on Convex and Compact Sets}

This section give applications of the preceding statements. In this sense, the first purpose is to present some of applicattions of our former Maximal Principles on convex and compact sets.

In connection with the preceding, let $B: X \rightarrow \mathbb{R}_{+}^{0}:=[0,+\infty)$ be a convex function such that $B((x+y) / 2) \geq B(x)$ for all $x, y \in X$, where $B$ is a lower semicontinuous function on convex and compact set $X$ in a linear topological space $Y$. Define a relation $\preccurlyeq B$,con on $X$ by the following conditions:

$$
\left\{\begin{array}{lcc}
x \preccurlyeq B, \text { con } y & \text { if and only if } & A(x, y) \leq B(y)-B(x), \\
x={ }_{B, \text { con }} y & \text { if and only if } & B(x)=B(y),
\end{array}\right.
$$

where $A(x, y):=B((x+y) / 2)-B(x) \geq 0$ and $A(x, y)=0$ iff $x=y$ for all $x, y \in X$. It is to verify that $\preccurlyeq B$,con is a partial ordering (asymmetric and transitive relation) in $X$. The set $X$, together with this partial ordering, is denoted by $X_{B, c o n}$.

Theorem 6.1. (Minimal Ordering Principle). Let $X$ be a convex and compact set in a linear topological space, with the poset $X_{B, c o n}$. Then $X_{B, \text { con }}$ has at least countable or finite minimal elements $z_{k} \in X_{B, \text { con }}$, with $z_{k} \preccurlyeq B$,con $x$ for given $x \in X_{B, c o n}$.

Proof. Let $C$ be a chain in $X_{B \text {,con }}$ and let $t \in C$ be given. Denote by $\beta$ the greatest lower bound of the set $\{B(x): x \in C\}$, i.e., $\beta:=\inf \{B(x): x \in C\}$. If $B(m)=\beta$ for some $m \in C$, then $m$ is a lower bound in $C$. For, if $x \preccurlyeq B$,con $m$ for some $x$ in $C \backslash\{m\}$, then $B(x) \leqslant B(m)$, which yields $B(x)<\beta$, which is a contradiction. Therefore, one can assume $B(x) \neq \beta$ for all $x \in C$. Then the set $M(x, n)$ of all $y \in C$ with $y \preccurlyeq B$,con $x$ and $\beta<B(x)<\beta+1 / n$ is nonempty for each $n \in\{1,2, \ldots\}:=\mathbb{N}$ and $x \in C$. In fact, there is a $y \in C$ satisfying $\beta<B(y)<\beta+1 / n$, and so $y$ belongs to $M(x, n)$ if $y \preccurlyeq B$,con $x$; if $x \preccurlyeq B$,con $y$ then since $B(x) \leqslant B(y)$, we have $\beta<B(x) \leq B(y)<\beta+1 / n$, which shows that $x$ belongs to $M(x, n)$. Let $I$ be a choice function for the family of all nonempty subsets of $C$. Then, by the recursion theorem, there is a sequence $\left\{x_{n}\right\}_{n \in \mathbb{N}}$ in $C$ such that $x_{0}=t$ and $x_{n+1}=I\left(M\left(x_{n}, n\right)\right)$ for $n \in \mathbb{N}$. Hence the compactness implies that $\left\{x_{n}\right\}_{n \in \mathbb{N}}$ has a convergent subsequence $\left\{x_{n(k)}\right\}_{k \in \mathbb{N}}$ to $\xi \in X$.

Now let $x$ be in $C$. Then we can find an $i \in \mathbb{N}$ such that $B\left(x_{i}\right)<\beta+1 / i<B(x)$. Since $x$ and $x_{i}$ are in the chain $C$, this implies $x_{i} \preccurlyeq B$,con $x$. Therefore, we have $B\left(x_{i}\right) \leqslant B(x)$, for every $n \geqslant i$. Thus, by lower semicontinuity of $B$, we obtain $B(\xi) \leqslant B(x)$, i.e., $\xi \preccurlyeq B$, con $x$. This shows that $\xi$ is a lower bound of $C$. By the nature of $C$ (by the dual form of Lemma of Infinite Maximality) it follows that $X_{B, \text { con }}$ has at least countable or finite minimal elements in $X_{B, c o n}$. This completes the proof.

As an immediate application of the preceding statement (Minimal Ordering Principle) we have the following extension and a new solution of Schauder's 54th problem in Scottish Book on linear topological spaces. 
Theorem 6.2. (Extension of Schauder's 54th problem). Let $C$ be a nonempty convex compact subset of a linear topological space $X$ and suppose that $T: C \rightarrow C$ is a continuous mapping. Then $T$ has at least countable or finite fixed points in $C$.

Proof. Consider the partially ordered set $C_{B, c o n}$ and let $\xi_{k}$ be minimal elements. Using the equation $x=T(x)$, it also follows from (Co) that we have $T(x) \preccurlyeq_{B \text {,con }} x$ for all $x \in C$ and $T \xi_{k} \preccurlyeq_{B \text {,con }} \xi_{k}$ in $C_{B \text {,con }}$ and, because, $\xi_{k}$ are minimal, it follows that $\xi_{k}={ }_{B \text {,con }} T\left(\xi_{k}\right)$. Thus, from (Co) we have $B\left(\xi_{k}\right)=B\left(T \xi_{k}\right)$, i.e., $\xi_{k}=T\left(\xi_{k}\right) \in C$. This completes the proof.

In connection with this statement, also as a direct consequence of Theorem 6.1 (Minimal Ordering Principle), we obtain for fixed apices the following result.

Theorem 6.3. (Form of Schauder's 54th problem for fixed apices). Let $C$ be a nonempty convex compact subset of a linear topological space $X$ and suppose that $T: C \rightarrow C$ is a continuous mapping. Then $T$ has at least countable or finite fixed apices in $C$.

Proof. Consider the partially ordered set $C_{B, c o n}$ and let $\xi_{k}$ be minimal elements. Using the equation $x=T^{2}(x)$, it also follows from (Co) that we have $T^{2}(x) \preccurlyeq_{B \text {,con }} x$ for all $x \in C$ and $T^{2}\left(\xi_{k}\right) \preccurlyeq B$,con $\xi_{k}$ in $C_{B \text {,con }}$ and, because, $\xi_{k}$ are minimal, it follows that $\xi_{k}={ }_{B, c o n} T^{2}\left(\xi_{k}\right)$. Thus, from (Co) we have $\xi_{k}=T^{2}\left(\xi_{k}\right)$, i.e., from the preceding facts on apices in part $1, T$ has at least countable or finite fixed apices. The proof is complete.

On the other hand, let $B: X \rightarrow \mathbb{R}_{+}^{0}$ be a convex function such that we have $B((x+y) 2) \geq B(x)$ for all $x, y \in X$, where $B$ is a continuous (or a lower semicontinuous) function on convex set $X$ in a linear topological space $Y$. Define a relation $\preccurlyeq_{B \text {,con }}$ with (Co). It is verify that $\preccurlyeq_{B \text {,con }}$ is a partial ordering (asymmetric and transitive relation) in $X$. The convex set $X$, together with this partial ordering, is denoted by $X_{\text {con }, B}$.

As an immediate consequence of the preceding proof of Theorem 6.1 (Minimal Ordering Principle) we have direct the following fact!

Proposition 6.1. Let $X$ be a convex set in a linear topological space $Y$, then there exists a continuous (or a lower semicontinuous) function $G: X \rightarrow \mathbb{R}_{+}^{0}$ such that $T: X \rightarrow X$ with the property $(F)$.

Also, let $X$ be a Banach space and let $Y:=(Y, \preccurlyeq)$ be an ordered Banach space. Suppose that $B: X \rightarrow Y$ is a convex operator ${ }^{10}$ such that $\theta \preccurlyeq$

${ }^{10}$ Convex operators. Let $X$ and $Y$ be real ordered Banach spaces with order $\preccurlyeq$, and let $T: D(T) \subset X \rightarrow Y$ be an operator. $T$ is convex iff $D(T)$ is a convex set such that with $x \prec y$ the following inequality holds

$$
T(\lambda x+(1-\lambda) y) \preccurlyeq \lambda T(x)+(1-\lambda) T(y)
$$

for all $x, y \in D(T)$ and for arbitrary $\lambda \in(0,1)$. The operator $T$ is concave iff $-T$ is a convex operator. 
$B((x+y)-B(x))$ for all $x, y \in X$, where $B$ is a continuous operator. Define a relation $\preccurlyeq_{B, o p r}$ on $X$ by the following conditions:

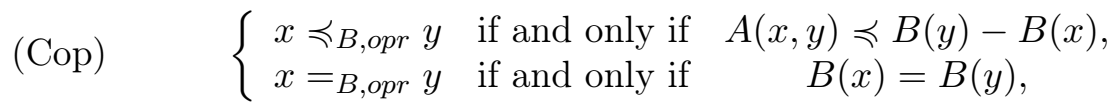

where $A(x, y):=B((x+y) / 2)-B(x) \succcurlyeq \theta$ and $A(x, y)=\theta$ iff $x=y$ for all $x, y \in X$. It is to verify that $\preccurlyeq_{B, o p r}$ is a partial ordering (asymmetric and transitive relation) in $X$. The set $X$, together with this partial ordering, is denoted by $X_{B, o p r}$.

Theorem 6.4. (Minimal Principle). Let $X$ be a convex and compact set in a linear topological space, with the poset $X_{B, o p r}$. Then $X_{B, \text { opr }}$ has at least countable or finite minimal elements $z_{k} \in X_{B, o p r}$, with $z_{k} \preccurlyeq B$, opr $x$ for given $x \in X_{B, \text { opr }}$.

The proof of this statement is a total analogy with the proof of Theorem 4.1. Thus the proof we omit.

As an immediate application of the preceding statement (Minimal Principle) we obtain the following result on linear topological spaces, as Theorem 5.2 in the different form.

Theorem 6.5. Let $C$ be a nonemty convex compact subset of a linear topological space $X$, and suppose that $T: C \rightarrow C$ is a continuous mapping. Then $T$ has at least countable or finite fixed points in $C$.

Proof. (Different of the proof of Theorem 5.2). Consider the partially ordered set $C_{B, o p r}$ and let $\xi_{k}$ be minimal elements. Using the equation $x=T(x)$, it is also follows from (Cop) that we obtain $T(x) \preccurlyeq B$, opr $x$ for all $x \in C$ and $T\left(\xi_{k}\right) \preccurlyeq_{B, o p r} \xi_{k}$ in $C_{B, o p r}$ and, because, $\xi_{k}$ are minimal, it follows that $\xi_{k}={ }_{B, o p r} T\left(\xi_{k}\right)$. Thus, from (Cop) we have $B\left(\xi_{k}\right)=B\left(T\left(\xi_{k}\right)\right.$ ), i.e., $\xi_{k}=T\left(\xi_{k}\right) \in C$. This completes the proof.

Annotation. We notice that the proof of Theorem 6.3 (for fixed apices), also, we can given via the partially ordered set $C_{B, o p r}$. The proof is an analogy with the given proof of Theorem 6.3. The proof we omit.

We are now in a position to formulate our following known applications. In this sense we obtain three fundamental famous principles of Brouwer, Banach and Schauder.

First, as an immediale and direct application of Theorem 6.1 (Minimal Ordering Principle) and Theorem 6.3 we obtain an extension of Brouwer's theorem in the following two forms.

Theorem 6.6. (Fixed Apices). Suppose that $C$ is a nonemty convex compact subset of $\mathbb{R}^{n}$, and that $T: C \rightarrow C$ is a continuous mapping. Then $T$ has at least countable or finite fixed apices.

Theorem 6.7. (General Brouwer Theorem). Suppose that $C$ is a nonemty convex, compact subset of $\mathbb{R}^{n}$, and that $T: C \rightarrow C$ is a continuous mapping. Then $T$ has at least countable or finite fixed points in $C$. 
In this sense, as a direct consequence of Theorem 6.7, we obtain the following well-known Brouwer's theorem.

Theorem 6.8. (Brouwer, [1912]). Suppose that $C$ is a nonempty convex, compact subset of $\mathbb{R}^{n}$, and that $T: C \rightarrow C$ is a continuous mapping. Then $T$ has a fixed point in $C$.

Proof. Since $C$ is a convex and compact subset of a Banach space, from Theorem 6.1 (Minimal Ordering Principle) and Theorem 6.7, we obtain this statement.

Let $X, Y$ be topological spaces. A continuous map $F: X \rightarrow Y$ is called compact if $F(X)$ is contained in a compact subset of $Y$. If $X$ and $Y$ are Banach's spaces and $T: D(T) \subset X \rightarrow Y$, then $T$ is called compact if $T$ is continuous and $T$ maps bounded sets into relatively compact sets. Compact operators play a central role in nonlinear functional analysis. Schauder's theorem is a generalization of Brouwer's theorem to infinite dimensional normed linear spaces, with the preceding fact.

We can now formulate Brouwer's theorem in a manner valid for all normed linear spaces.

Theorem 6.9. (Schauder, [1927]). Let $C$ be a nonempty, closed, bounded, convex subset of the Banach space $X$, and suppose $T: C \rightarrow C$ is a compact operator. Then $T$ has a fixed point $\xi \in C$.

We also have an alternate version of the preceding Schauder fixed point theorem. Further we give a proof, via Theorem 6.1 (Minimal Ordering Principle) and Theorem 6.2 , of this fact.

Theorem 6.10. (Schauder, [1930]). Let $C$ be a nonempty, compact, convex subset of a Banach space $X$, and suppose $T: C \rightarrow C$ is a continuous operator. Then $T$ has a fixed point in $C .{ }^{11}$

${ }^{11}$ Schauder biography. In 1978 Władisław Orlicz briefed: The Polish mathematician Juliusz Schauder was born in 1899 in Lwow. He studied at the University of Lwow under Banach, who together with Hugo Steinhaus created the Polish functional analytic school in the twenties in Lwow. Personal contact with Lean Lichtenstein awakened Schauder's interest in differential equations. After receiving a Rockefeller grant in 1932, he spent some time in Leipzig with Lichtenstein. In this period he also stayed in Paris, working together with Jean Leray. The fruit of this collaboration was the fundamental work: "Topologie et équations fonctionelles" which appeared in 1934. In 1938, Schauder and Leray received the Grand Prix International of Metaxas.

After the occupation of Lwow by the German army in June 1941, Schauder, like all others of Jewish descent, became a victim of Hitler's persecution. He was forced into hiding together with his wife and small daughter, under an assumed name. In 1943, during an extermination action, he, and shortly thereafter his wife too, was murdered by the German fascists. His daughter survived.

The essential character trait of Schauder was his extreme passion for mathematics, which united him with his teacher Stefan Banach. 
We notice that this statement is a direct translation of the Brouwer fixed point theorem to Banach spaces.

Proof. Since $C$ is a convex and compact subset of Banach space, from Theorem 6.1 (Minimal Ordering Principle) and Theorem 6.2, we obtain this statement.

Theorem 6.11. (General Schauder Theorem). Let $C$ be a nonempty, compact, convex subset of a Banach space $X$, and suppose $T: C \rightarrow C$ is a continuous operator. Then $T$ has at least countable or finite fixed points in C.

This statement is a direct consequence of Theorem 6.1 (Minimal Ordering Principle) and Theorem 6.2. Also, the following result is a direct consequence of Theorem 6.1 (Minimal Ordering Principle) and Theorem 6.3.

Theorem 6.12. (Fixed Apices). Let $C$ be a nonempty, compact, convex subset of a Banach space $X$, and suppose $T: C \rightarrow C$ is a continuous operator. Then $T$ has at least countable or finite fixed apices in $C$.

The following statement provides an important example of $A$-variation principle. We notice that this statement plays an important role in a fixed point theory.

Proposition 6.2. (Browder [1965], Göhde [1965], Kirk [1965]). Suppose that the map $T: M \subset X \rightarrow M$ is nonexpansive, where $M$ is a nonempty, closed, bounded, and convex set in the uniformly convex Banach space $X$. Then the fixed point set of $T, \operatorname{Fix}(T)$, is nonempty, closed, and convex.

Proof. Since $M$ is a convex subset of Banach space $X$, from the proof of Theorem 6.1 (Minimal Ordering Principle), we have that $T: M \rightarrow M$ is $A$-variation, where $A(x, y):=B((x+y) / 2)-B(x)$. The set $M$ is closed in $X$, and thus $M$ is a complete space. It is easy to see that $T$ satisfies all the required hypotheses in Theorem 5.1. Hence, it follows from the Theorem 5.1 that $T$ has a fixed point in $M$. Also, the set Fix $(T)$ is closed and convex by the preceding. The proof is complete.

Further, as an immediate fact of Theorem 6.1 (Minimal Ordering Principle) and Theorem 6.2, we have on metric spaces the following essential result.

Proposition 6.3. (Tasković, [1993]). Let D be a metric convex subset in metric space $(X, \rho)$ with the property of TCS-convergence. If $T: D \rightarrow D$ is a continuous mapping (or $x \mapsto \rho(x, T(x))$ is a lower semicontinuous mapping), then $T$ has a fixed point in $X$.

\section{Solution of Schauder's 54th Problem in Scottish Book ${ }^{12}$}

\footnotetext{
${ }^{12}$ History of Schauder's problem. The most famous of many open problems in nonlinear analysis is Schauder's problem (in Scottish book, problem 54). For some answers on
} 
Introduction and history. The most famous of many problems in nonlinear analysis is Schauder's problem (Scottish book, problem 54) of the following form, that if $C$ is a nonempty convex compact subset of a linear topological space does every continuous mapping $f: C \rightarrow C$ have a fixed point?

The answer I gives in this part that is yes, as first time by Tasković in 1998. In this connection this part proves and extends the Markoff-Kakutani theorem to arbitrary linear topological space as an immediate consequence of the preceding solution of Schauder's problem.

During the last twenty years this old conjecture was intensively examined by many mathematicians. For sets in normed spaces this has been proved by Schauder and for sets in locally convex spaces by Tychonoff.

In this part we prove that if $C$ is a nonempty convex compact subset of a linear topological space, then every continuous mapping $f: C \rightarrow C$ has at least countable or finite fixed points.

Brouwer's theorem of fixed point is one of the oldest and best known results in mathematics. Schauder's theorem of fixed point is a generalization of Brouwer's theorem to infinite dimensional normed linear spaces. Schauder's theorem states that every continuous mapping of a compact convex subset of a normed linear space into itself has a fixed point.

Schauder's problem (Scottish book, problem 54) is in the following form: Does every continuous mapping $f: C \rightarrow C$ of a nonempty convex compact subset $C$ in arbitrary linear topological space have a fixed point?

For locally convex space the answer is yes from Tychonoff [1935]. Namely, in 1935, Tychonoff had shown that if $C$ is a nonempty convex compact subset of a locally convex space, then every continuous map $f: C \rightarrow C$ has a fixed point.

Schauder's theorem was further extended by Kukuhara [1950], Mazur [1938], Gohde [1965], Fan [1961], Dugundji [1976], Granas [1957], Klee [1960], Kirk [1965], Idzik [1988], Riedrich [1976], Eisenack-Fenske [1978], Jahn [1984], Browder [1965], Darbo [1955], Delenau [1961], Sadovskij [1967], Krasnoselskij [1955], Reinermann [1971], Tasković [1993], and many others.

The literature on applications of Schauder's theorem to nonlinear problems is extensive. The first result was proved by Markoff [1936] with the aid of the Schauder-Tychonoff fixed point theorem.

Kakutani [1941] found a direct elementary proof of the Markoff theorem. Extensions of Markoff-Kakutani theorem is due to Day [1961], Hahn [1978] and Ryll-Nardzewski [1966].

In this part we give the complete solution and an extension of the preceding well known Schauder's problem of fixed point by Tasković [1998]. Also, this solution is answering a question of S. Ulam. In connection with this, in this part, we extend the Markoff-Kakutani theorem to arbitrary linear topological spaces as an immediate consequence of the preceding Tasković's solution in 1998 as and a new solution of Schauder's problem.

this problem see papers of: Tychonoff, Fréchet, Leray, Borsuk, Steinhaus, Mazurkiewicz, Kuratowski, Knaster, Krasnoselskij, Ky Fan, Klee, Caristi, Kirk, Browder, Dugundji, Granas, and many others.

J. Schauder himself set down this problem in 1927 and 1930 respectively and had it published in: Math. Zaitschrift and Studia Mathematica. The problem gained the importance when it was put forward by StefanBanach in 1930 at the World congress of mathematicians in Moscow.

First positive answer for locally convex space was given by Tychonoff in 1935. It was J. Schauder who presented (personally) Tychonoff's paper in Zbl. für Math. 12 (1936), with number 308. 
Answer to Schauder's problem is affirmative. From the preceding statements and some further facts we are now in the position to formulate the following solution which is, also, an extension of Schauder problem.

Theorem 7.1. (Answer is yes for Schauder's problem). Let $C$ be a nonempty convex compact subset of a linear topological space $X$ and suppose that $T$ : $C \rightarrow C$ is a continuous mapping. Then $T$ has a fixed point in $C$.

This result is a special case of the former Theorem 6.2. Thus the proof of this state ment we omit. For the first proof of Theorem 7.1 see Tasković [1998].

Annotation. Let us emphasize that the above solution for the Schauder's problem was presented by Tasković in 1998. Another different solution for the same problem was published by R. Cauty in 2001, three years later after M. R. Tasković. See: Tasković [1998] and Cauty [2001]! Also see: Rus [1999].

Some further applications. As an immediate corollary of the preceding solved problem (Theorem 7.1), we obtain one of the basic results in nonlinear functional analysis which is an extension of the Markoff-Kakutani theorem.

Theorem 7.2. Let $C$ be a nonempty convex compact set in a linear topological space $X$ and let $\mathcal{F}$ be a commuting family of continuous affine maps of $C$ into itself. Then $\mathcal{F}$ has a common fixed point in $C$.

A brief proof of this result based on Theorem 7.1 may be found in Tasković: [1998], [2001]. Also see Tasković [2005].

Proof. Let $\operatorname{Fix}(T)$ be a fixed point set of a map $T$. By Theorem 7.1, $\operatorname{Fix}(T)$ is a nonempty set for each $T \in \mathcal{F}$. Moreover, $\operatorname{Fix}(T)$ is compact being closed in the compact set $C$, and $\operatorname{Fix}(T)$ is convex because $T$ is affine.

We must prove that $\cap\{\operatorname{Fix}(T): T \in \mathcal{F}\}$ is a nonempty set; because each set $\operatorname{Fix}(T)$ is compact, it is sufficient to show that each finite intersection

$$
\operatorname{Fix}\left(T_{1}, \ldots, T_{n}\right):=\bigcap_{i=1}^{n} \operatorname{Fix}\left(T_{i}\right)
$$

is nonempty. We proceed by induction on the number $n \in \mathbb{N}$ of $T_{i}$, the result being true for $n=1$. Assume that $\operatorname{Fix}\left(T_{1}, \ldots, T_{n}\right)$ is nonempty whenever $i<n$ and consider any $n$ members $T_{1}, \ldots, T_{n}$ of $\mathcal{F}$. Because $\mathcal{F}$ is commuting, we find that

$$
T_{n}\left[\operatorname{Fix}\left(T_{1}, \ldots, T_{n-1}\right)\right] \subset \operatorname{Fix}\left(T_{1}, \ldots, T_{n-1}\right),
$$

for if $x \in \operatorname{Fix}\left(T_{1}, \ldots, T_{n-1}\right)$ then $T_{i}\left[T_{n}(x)\right]=T_{n}\left[T_{i}(x)\right]=T_{n}(x)$ for each $i<n$ so $T_{n}(x) \in \operatorname{Fix}\left(T_{1}, \ldots, T_{n-1}\right)$.

Since $\operatorname{Fix}\left(T_{1}, \ldots, T_{n-1}\right)$ is a nonempty compact convex set, we conclude from Theorem 7.1 that $\operatorname{Fix}\left(T_{1}, \ldots, T_{n}\right)$ is a nonempty set. This completes the induction and the proof.

As a direct application of the preceding Theorem 7.2 we obtain the following well known result for families of mappings. 
Theorem 7.3. (Markoff [1936], Kakutani [1938]). ${ }^{13}$ Let $C$ be a compact convex set in a locally convex linear space $X$, and let $\mathcal{F}$ be a commuting family of continuous affine maps of $C$ into itself. Then $\mathcal{F}$ has a common fixed point.

We notice that Markoff-Kakutani theorem has numerous applications. An example, Kakutani [1938] has proved that Theorem 7.3 implies Hahn-Banach theorem. A brief proof of Theorem 7.3 may be found in: Dugundji-Granas [1982], Istratescu [1981], Zeidler [1985], and Tasković [1993].

Some annotations. An extension of Theorem 7.3 was discovered by Ryll-Nardzewski [1966] who based the proof on probabilistic arguments. The first geometric proof of this extension was given by Namioka-Asplund [1967]. A special case of this extension was found by $\mathrm{Hahn}$ [1967]. For this also see: Hansel-Troallic [1976].

Further applications. As an immediate application of $A$-variation principle (Theorem 5.1) we have Tychonoff's theorem on locally convex spaces in the following form. This result of Tychonoff is also a special case of Theorem 7.1 .

Theorem 7.4. (Tychonoff, [1935]). ${ }^{14}$ Let D be a compact convex subset in locally convex linear space $E$. Then every continuous mapping $f$ of $D$ into itself has a fixed point in $D$

Proof. Since $D$ is a convex subset in locally convex space $E$, from Proposition 5.1, we have that $f: D \rightarrow D$ is $A$-variation. The set $D$ is compact in $E$, and thus $D$ satisfies the condition of TCS-convergence. Since $f$ is also a continuous mapping, hence all the required hypotheses in Theorem 5.1 hold. Applying Theorem 5.1 we obtain that $f$ has a fixed point in $D$. The proof is complete.

${ }^{13}$ History of Markoff theorem. Theorem 7.3 was proved by Markoff [1936] with the aid of the Schauder-Tychonoff fixed point theorem. Kakutani [1938] found a direct elementary proof of Theorem 7.3 (valid in any linear topological space not necessarily locally convex), and demonstrated the importance of the result by giving a number of applications; he also showed that Theorem 7.3 implies the Hahn-Banach theorem in linear functional analysis. The following result (as an extension) is due to Day [1961] in the following form: Let $K$ be a convex compact set in a locally convex space and let $S$ be a left-amenable semigroup of continuous affine maps acting on $K$. Then there is a common fixed point under $S$.

Because every abelian semigroup of left-amenable and since in the formulation of Theorem 7.3 a commutating family can clearly be replaced by an abelian semigroup, the result of Day contains the Markoff-Kakutani theorem as a special case.

${ }^{14}$ Schauder's report. The paper of Tychonoff [1935] is to present in Zbl. für Math. 12 (1936) with number 308 personal J. Schauder who briefs: "On generalizing a reviewer's fixpoint theorem the proof of which yields the existence of a fixpoint only when the space is linear, metric, and locally convex (J. Schauder, Studia Math. 2 (1930), 171-180, Theorem I) the author proved the following...". 
Annotation. We notice that a different proof of the preceding Theorem 7.4 can be given with Theorem 7.1. In this sense a brief proof of this may be found in Tasković [2001].

Theorem 7.5. (Mazur [1938], Hukuhara [1950]). ${ }^{15}$ Let D be a convex subset in a locally convex space $E$ and let $f: D \rightarrow D$ be a compact mapping. Then $f$ has a fixed point in $D$.

Proof. Since $D$ is a convex subset in $E$, from Proposition 5.1, we have that $f$ is an $A$-variation mapping. Also, it is easy to see that $f$ satisfies all the required hypothesis in Theorem 5.1. Hence, it follows from Theorem 5.1 that $f$ has a fixed point in $D$.

On the other hand, as an immediate consequence of Theorem 5.1, we obtain the following geometrical fact on fixed points.

Theorem 7.6. Let $T$ be a self-map on a topological space $X$ and $A: X \times$ $X \rightarrow \mathbb{R}_{+}^{0}$ be a function with properties: $A(a, b)=0$ iff $a=b$ and $A(a, c) \leq$ $A(a, b)+A(b, c)$ for all $a, b, c \in X$. Suppose that there exists a continuous function $G: X \rightarrow \mathbb{R}_{+}^{0}$ such that

$$
A(x, T x) \leqslant|G(x)-G(T x)|
$$

for every $x \in X$. If $X$ satisfies the condition of TCS-convergence and if $b \mapsto A(a, b)$ is continuous, then $T$ has a fixed point $\xi \in X$.

A brief proof of this statement, based on Theorem 5.1, may be first found in Tasković [1998]. In connection with this also see: Tasković [2005].

\section{Fredholm and Leray-Schauder alternatives}

In this section, first, we apply the technics of maximal elements to the equation $x=\lambda f(x)$ for some $0<\lambda \leq 1$, where $f$ is a compact or completely continuous operator; first we have from Theorem 6.1 the following ordered principle.

Theorem 8.1. (Ordering Principle). $\quad$ Let $C$ be a convex set in a linear topological space $Y$, with the poset $C_{B, c o n}$. If $f: C \rightarrow C$ is a compact or a completely continuous operator, then $C_{B, \text { con }}$ has at least countable or finite minimal elements $z_{k} \in C_{B \text {,con }}$, with $z_{k} \preccurlyeq B$,con $x$ for given $x \in C_{B, \text { con }}$.

${ }^{15}$ Fixed points in locally convex spaces. The main results in the text extend to locally convex spaces and also to some spaces which are not locally convex. Topological transversality can be proved without local convexity from Granas [1976]. The fact that convex sets in locally convex spaces have such a property was proved by $\mathrm{Hukuhara}$ [1950].

The antipodal theorem of Borsuk was generalized to locally convex spaces by Altman [1958a]. Also, Leray [1950] extended to locally convex spaces the invariance of domain theorem and applied it to establish in such spaces the Fredholm alternative; another proof of invariance of domain similar to that given in the text and based on Borsuk's theorem will be found in Altman [1958a]. For more details of this see: Klee [1960a] and Hahn [1978]. 
As an immediate application of the preceding statement we obtain the following extension of Leray-Schauder and Fredholm alternatives.

Theorem 8.2. Let $C$ be a nonempty convex subset of a Banach space $X$ and let $f: C \rightarrow C$ be a compact or a completely continuous mapping. Then the equation

$$
x=\lambda f(x) \text { for some } 0<\lambda \leq 1,
$$

has at least countable or finite solutions in $C$.

Proof. Consider the partially ordering set $C_{B, c o n}$ and let $\xi_{k}$ be minimal elements. Using (LŠ) it also follows from (Co) that we obtain $\lambda f(x) \preccurlyeq B$,con $x$ for all $x \in C$ and $\lambda f\left(\xi_{k}\right) \preccurlyeq B$,con $\xi_{k}$ in $C_{B, c o n}$ and, because $\xi_{k}$ are minimal, it follows that $\xi_{k}={ }_{B, \text { con }} \lambda f\left(\xi_{k}\right)$. Thus, from (Co) we have $B\left(\xi_{k}\right)=B\left(\lambda f\left(\xi_{k}\right)\right)$, i.e., $\xi_{k}=\lambda f\left(\xi_{k}\right) \in C$. The proof is complete.

Theorem 8.3. Let $C$ be a nonempty convex subset of a Banach space $X$ and let $f: C \rightarrow C$ be a compact or completely continuous mapping. Then the equation

$$
x=\lambda f^{2}(x) \text { for some } 0<\lambda \leq 1,
$$

has at least countable or finite solutions in $C$.

The proof of this statement is a total analogy with the proof of Theorem 8.2. Thus, this proof we omit.

Annotations. If (L $\check{S}$ ) only holds for some $0<\lambda<1$, then the equation in the following form as

$$
x=\lambda f(x) \text { for some } 0<\lambda<1
$$

has at least countable solutions or the equation $x=f(x)$ has at least finite solutions.

Indeed, if the set solutions of (L $\check{S}$ ) denoted by $\mathcal{G}$, is bounded, then $f \mid C \cap$ $B(0, r): C \cap B(0, r) \rightarrow C$ is a compact map, and no $x \in \partial[C \cap B(0, r)]$ can satisfy the property: there is an $x \in C \cap B(0, r)$ with $x=\lambda f(x)$ for some $0<\lambda<1$ (see: Dugundji-Granas [1982, Theorem 5.1, p. 61].). This means that, in the second case of finitess, $f: C \rightarrow C$ has at least finite fixed points. In this sense, Theorem 8.2 applied to compact or completely continuous operators yields.

Theorem 8.4. (The Leray-Schauder alternative). ${ }^{16}$ Let $C$ be a convex subset of a Banach space $Y$, and assume $0 \in C$. Let $f: C \rightarrow C$ be a completely

${ }^{16}$ The Work of Leray and Schauder. As long ago as 1934 a novel approach towards fixed point theorems was developed and published by Leray and Schauder. Included in this investigation is the relationship between the solubility of an equation of the type $x-f(x)=0$ and the topological degree at $y=0$ of the mapping

$$
x \mapsto y=x-f(x)
$$

and the invariance of the topological degree, under continuous deformation of the map involved. This program was carried out under the assumption that $f$ is defined and 
continuous operator and let

$$
\mathcal{E}(f):=\{x \in C: x=\lambda f(x) \text { for some } 0<\lambda<1\},
$$

then either $\mathcal{E}(f)$ is unbounded set or $f$ has at least finite fixed points in $C$.

In connection with the preceding, also, we apple the technics of maximal elements (Theorem 8.2) to the equation $y=x-f(x)$. We have, first, the following result.

Theorem 8.5. Let $C$ be a nonempty convex subset of a Banach space $X$ and let $f: C \rightarrow C$ be a compact or a completely continuous mapping. Then the equation

$$
z=x-f(x) \quad \text { for every } z \in C,
$$

has at least countable or finite solutions in $C$.

Proof. Consider the partially ordered set $C_{B, o p r}$ and let $\xi_{k}$ be minimal elements. Using (Fr) it also follows from (Cop), for $B(t)=t$ and $z=x-y$, that we obtain solutions for the equation (Fr). The proof is complete.

Annotations. On the second condition, we can introduction on $C$, the following ordering: $x \preccurlyeq y$ if and only if $z=x-y$ for some $z \in C$, and $x=y$ if and only if $z=x-y$ for every $z \in C$. Applying Theorem 6.4 (Minimal Principle), in this case, we obtain Theorem 8.5.

Theorem 8.6. Let $C$ be a nonempty convex subset of a Banach space $X$ and let $f: C \rightarrow C$ be a compact or completely continuous mapping. Then the equation

$$
z=x-f^{2}(x) \quad \text { for every } z \in C,
$$

has at least countable or finite solutions in $C$.

The proof of this statement is a total analogy with the proof of Theorem 8.5. Thus the proof we omit.

As an immediate application of the preceding Theorem 8.5, direct, we obtain the following important result.

Theorem 8.7. (The Fredholm alternative). Let $E$ be an arbitrary normed space, and let $f: E \rightarrow E$ be a completely continuous linear operator. Then the equation

$$
z=x-f(x) \quad \text { for every } z \in E,
$$

has a unique solution, or the equation $0=x-f(x)$ has a nontrivial solution.

continuous on the closure of a bounded open set $Q$ in space and that $f(\bar{Q})$ is relatively compact in space.

Schauder and Leray succeeded in carrying out this program in such a way as to derive existence theorems for general types of partial differential equations, the second stage of the operation demanding a great deal of ingenuity and considerable prior knowledge of more specialized types of partial differential equations. 
On the other hand, as an immediate application of the preceding Theorem 8.6, direct, we obtain the following result.

Theorem 8.8. (Linear alternative). Let $E$ be an arbitrary normed space, and let $f: E \rightarrow E$ be a completely continuous linear operator. Then the equation

$$
z=x-f^{2}(x) \quad \text { for every } \quad z \in E,
$$

has a solution, or the equation $0=x-f^{2}(x)$ has a nontrivial solution.

\section{Generalized Peano's Theorem}

Further we give an application of General Schauder fixed point theorem to differential equations. As a parallel and contrast to the Picard-Lindelöf theorem we consider the initial value problem of the form as

$$
x^{\prime}(t)=f(t, x(t)), \quad x\left(t_{0}\right)=y_{0},
$$

on $\left[t_{0}-c, t_{0}+c\right]$. Geometrically, (5) means that we are looking for a curve which satisfies the differential equation and passes through $\left(t_{0}, y_{0}\right)$ as in the following Figure with $y_{0}=p_{0}$. At the end points $t=t_{0} \pm c$, where $x^{\prime}(t)$ is to be interpreted as the appropriate one-sided derivative.

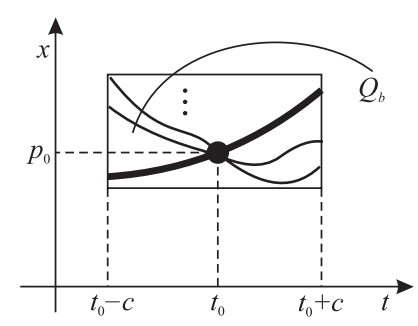

Proposition 9.1. (Peano [1890], Tasković [2012]). Let there be given real numbers $t_{0}$ and $y_{0}$, and the rectangle of the form as

$$
Q_{b}:=\left\{(t, x) \in \mathbb{R}^{2}:\left|t-t_{0}\right| \leq a,\left|x-y_{0}\right| \leq b\right\},
$$

where $a$ and $b$ are fixed positive numbers. Suppose that $f: Q_{b} \rightarrow \mathbb{R}$ is continuous and bounded with the following condition of the form as

$$
|f(t, x)| \leq K \quad \text { for all }(t, x) \in Q_{b},
$$

and fixed $K>0$. Set $c:=\min \{a, K / b\}$. Then the initial value problem (5) has at least countable or finite continuously differentiable solutions on $\left[t_{0}-c, t_{0}+c\right]$.

Proof. (Application of Theorem 6.11). In addition to initial value problem (5), we also consider the integral equation of the form as

$$
x(t)=y_{0}+\int_{t_{0}}^{t} f(s, x(s)) d s,
$$


and next write this as the operator equation $x=T(x)$, for $x \in M \subset X$, where $X:=C\left(\left[t_{0}-c, t_{0}+c\right]\right), M=\left\{x \in X:\left\|x-y_{0}\right\| \leq b, \quad\right.$ and $\|x\|=$ $\left.\max _{t_{0}-c \leq t \leq t_{0}+c}|x(t)|\right\}$. The set $M$ is closed, convex, and bounded in $X$; also from this it follows $T(M) \subset M$. Since the operator $T$ is compact,we have that Tasković fixed point theorem (Theorem 6.11) implies the existence at least countable or finite solutions $x=T(x), x \in M$. The proof is complete.

\section{EXISTENCE PRINCIPLE FOR SySTEMS OF EQUATIONS}

As a simple application of the General Brouwer fixed point theorem, we will prove an important existence statement for the system of the form as

$$
g_{i}(x)=0, \quad \text { for } \quad i=1, \ldots, n ;
$$

where $x=\left(\xi_{1}, \ldots, \xi_{n}\right) \in \mathbb{R}^{n}$. The key for solution of this problem is in the next boundary condition which we can briefly write in the following form as

$$
\sum_{i=1}^{n} g_{i}(x) \xi_{i} \geqslant 0 \text { for all } x \text { with }\|x\|=r .
$$

We notice that this existing problem will play a decisive role in the discussion of the Galerkin method for monotone operators.

Proposition 10.1. (Solution of system equations (6)). Let $C l K(0, r)=$ $\left\{x \in \mathbb{R}^{n}:\|x\| \leq r\right\}$ for fixed $r>0$ and $x \mapsto\|x\|$ a norm on $\mathbb{R}^{n}$. Let $g_{i}: C l K(0, r) \rightarrow \mathbb{R}$ be continuous for $i=1, \ldots, n$. If (7) is satisfied, then (6) has at least countable or finite solutions $x$ with $\|x\| \leqslant r$.

Proof. (Application of General Brouwer Theorem). Set $g(x)=\left(g_{1}(x), \ldots, g_{n}(x)\right)$ and suppose that $g(x) \neq 0$ for all $x \in \mathrm{Cl} K(0, r)$. Then define

$$
f(x)=-r g(x) /\|g(x)\|,
$$

and, now $f$ is continuous map of compact, convex set $\mathrm{Cl} K(0, r)$ into itself. By General Brouwer fixed point theorem (Theorem 6.7) there exist at least countable or finite fixed points $x=f(x)$. Taking norms, we see that $\|x\|=r$. Furthermore,

$$
\sum_{i=1}^{n} g_{i}(x) \xi_{i}=-r^{-1}\|g(x)\| \sum_{i=1}^{n} f_{i}(x) \xi_{i}=-r^{-1}\|g(x)\| \sum_{i=1}^{n} \xi_{i}^{2}<0,
$$

contrary to the preceding fact (7). Thus, we have that $g(x)=0$ for countable or finite $x \in \mathrm{Cl} K(0, r)$. The proof is complete.

\section{An Extension of Leray-Schauder Principle}

We will now show how to use topological methods, and in particular, the General Schauder fixed point theorem for continuation with respect to $\lambda$ parameter. In this sense we have the following Leray-Schauder Principle in the form such as. 
Theorem 11.1. (Leray-Schauder [1934], Tasković [2005]). Let $X$ be a Banach space. Suppose that the operator $T: X \rightarrow X$ is compact and that there exists an $r>0$ such that

$$
x=\lambda T(x) \text { with } 0<\lambda<1 \text { implies }\|x\| \leq r,
$$

then the equation of the form as $x=T(x)$ has at least countable or finite solutions. (Notice that (8) is trivially fulfilled if the following inequality holds as $\left.\sup _{x \in X}\|T(x)\|<\infty\right)$.

Proof. (Application of Tasković theorem 6.11). Let $X$ be a Banach space and $T$ is a compact operator. We define an operator

$$
S(x)=\left\{\begin{array}{l}
T(x) \quad \text { if } \quad\|T(x)\| \leq 2 r, \\
\frac{2 r T(x)}{\|T(x)\|} \quad \text { if } \quad\|T(x)\|>2 r
\end{array}\right.
$$

and we claim that $S: M \rightarrow M$ is compact on $M:=\{x \in X:\|x\| \leq 2 r\}$. Obviously, $S$ is continuous. To establish compactness, let $\left\{x_{n}\right\}_{n \in \mathbb{N}}$ be a sequence in $M$. We consider two cases, namely there is: (a) a subsequence $\left\{y_{n}\right\}_{n \in \mathbb{N}}$ of $\left\{x_{n}\right\}_{n \in \mathbb{N}}$ such that $\left\|T\left(y_{n}\right)\right\| \leq 2 r$ for all $n \in \mathbb{N}$, and (b) a subsequence $\left\{y_{n}\right\}_{n \in \mathbb{N}}$ such that $\left\|T\left(y_{n}\right)\right\|>2 r$ for all $n \in \mathbb{N}$.

In case (a), the compactness of $T$ implies that there is a subsequence $\left\{z_{n}\right\}_{n \in \mathbb{N}}$ of $\left\{y_{n}\right\}_{n \in \mathbb{N}}$ such that $S\left(z_{n}\right)=T\left(z_{n}\right) \rightarrow y$ as $n \rightarrow \infty$.

In case (b), one can choose $\left\{z_{n}\right\}_{n \in \mathbb{N}}$ so that $1 /\left\|T\left(z_{n}\right)\right\| \rightarrow \alpha$ and $T\left(z_{n}\right) \rightarrow$ $y$ as $n \rightarrow \infty$ for suitable $\alpha$ and $y$, so that $S\left(z_{n}\right) \rightarrow 2$ ray as $n \rightarrow \infty$.

The Tasković fixed point theorem (Theorem 6.11) provides us with at least countable or finite $x \in M$ for which $S(x)=x$. If $\|T(x)\| \leqslant 2 r$, then $T(x)=S(x)=x$. The other case, ||$T(x) \mid>2 r$, is impossible, for otherwise,

$$
S(x)=\lambda T(x)=x, \quad 0<\lambda=\frac{2 r}{\|T(x)\|}<1,
$$

which forces $\|x\|=2 r$, while the condition (8) requires that $\|x\| \leqslant r$. The proof is complete.

Annotations. We notice that by using Theorem 11.1 we can obtain an existence proof for the stationary Navier-Stokes equations (i.e., stationary movement of a viscous fluid).

\section{Global alternative $n$-Person Games}

The game theory is a mathematical search for the optimal balance of conflicting interests, such as between two partners. As such, it is applicable to a wide variety of situations: social games, economic sompetetion between organizations, conflicts in nature, and so on. In former, the optimal strategies for both partners turn out to be described by saddle points in 1928 of John von Neumann.

Let $P:=(P, \preccurlyeq)$ be a partially ordered set by the ordering relation $\preccurlyeq$. The function $g: P^{k} \rightarrow P$ ( $k$ is a fixed positive integer) is decreasing on the 
ordered set $P$ if $a_{i}, b_{i} \in P$ and $a_{i} \preccurlyeq b_{i}(i=1, \ldots, k)$ implies $g\left(b_{1}, \ldots, b_{k}\right) \preccurlyeq$ $g\left(a_{1}, \ldots, a_{k}\right)$.

Let $L$ be a lattice and $g$ a mapping from $L^{2}$ into $L$. For any $g: L^{2} \rightarrow L$ it is natural to consider the following property of local comparability, which means, if $\xi \in L$ is comparable with $g(\xi, \xi) \in L$ then $\xi$ is comparable with every $t \in L$.

In this section, the optimal strategies for both partners turn out to be described by the equalities, whose existence we established in Tasković [2005]. For example in the following:

Let $P:=(P, \preccurlyeq)$ be a totally ordered set and let $g: P^{2} \rightarrow P$ be a decreasing mapping. We consider two players, $A$ and $B$. Players $A$ and $B$ have available sets of strategies $X \subset P$ and $Y \subset P$, respectively. Each point $x \in X$ and $y \in Y$ represents a possible choice by $A$ and $B$, respectively. If $A$ chooses $x$, and $B$ chooses $y$, then the function $(x, y) \mapsto \max \{x, y, g(x, y)\}$ represents the gain by $A$ and the function $(x, y) \mapsto \min \{x, y, g(x, y)\}$ represents the gain by $B$. The point $\xi \in P$ is called an optimal strategy if the following equality holds

$$
\xi:=\max _{x \in X, y \in Y} \min \{x, y, g(x, y)\}=\min _{x \in X, y \in Y} \max \{x, y, g(x, y)\} .
$$

In connection with this, we notice that the existence of the preceding optimal strategy is established in Tasković [2005].

In further, let $g: P^{k} \rightarrow P$ ( $k$ is a fixed positive integer) be a decreasing function and consider players $A_{1}, \ldots, A_{k}$ with sets of strategies $X_{1}, \ldots, X_{k}$ in $P$, respectively. Each point $\lambda\left(x_{1}\right) \in X_{1}, \ldots, \lambda\left(x_{k}\right) \in X_{k}$ for $x_{1}, \ldots, x_{k} \in$ $X$ where $X$ is a nonempty set represents a possible choice by $A_{1}, \ldots, A_{k}$; respectively. The object (point) $\xi \in P$ is called an $k$-optimal strategy, in this case, if the following equality holds

$$
\begin{aligned}
\xi & :=\max _{\lambda\left(x_{1}\right) \in X_{1}, \ldots, \lambda\left(x_{k}\right) \in X_{k}} \min \left\{\lambda\left(x_{1}\right), \ldots, \lambda\left(x_{k}\right), g\left(\lambda\left(x_{1}\right), \ldots, \lambda\left(x_{k}\right)\right)\right\}= \\
& =\min _{\lambda\left(x_{1}\right) \in X_{1}, \ldots, \lambda\left(x_{k}\right) \in X_{k}} \max \left\{\lambda\left(x_{1}\right), \ldots, \lambda\left(x_{k}\right), g\left(\lambda\left(x_{1}\right), \ldots, \lambda\left(x_{k}\right)\right)\right\} .
\end{aligned}
$$

In analogous with the preceding facts, the existence of the $k$-optimal strategy, in the preceding case, we established in Tasković [2005], i.e., if and only if the following equality holds in the form as

$$
\begin{aligned}
& \min \left\{\lambda_{1}\left(a_{1}\right), \ldots, \lambda_{k}\left(a_{k}\right), g\left(\lambda_{1}\left(a_{1}\right), \ldots, \lambda_{k}\left(a_{k}\right)\right)\right\}= \\
& =\max \left\{\lambda_{1}\left(a_{1}\right), \ldots, \lambda_{k}\left(a_{k}\right), g\left(\lambda_{1}\left(a_{1}\right), \ldots, \lambda_{k}\left(a_{k}\right)\right)\right\}
\end{aligned}
$$

for some $a_{1}, \ldots, a_{k} \in X$. (Intelligibly, in this case initial $X$ is a nonempty set. This is a game from the facts of chapter two of the book by Tasković [2005].) 
In connection with the preceding facts and results, if $L:=(L, \preccurlyeq)$ is a complete lattice with the local comparability, we have the following $n$-person game:

Interpretations. In connection with the preceding, the object (point) $\xi \in L$ in $(\mathrm{G})$ is called a global equilibrium object of the game.

In this sense, for $k=n$ we consider $n$-players $P_{1}, \ldots, P_{n}$. Let each player have available a strategy set consisting of a nonempty set $K_{i} \subset L(i=1, \ldots, n)$. We may regard the components of $p_{i} \in K_{i}(i=1, \ldots, n)$ for suitable $K_{i}$ as for the choices of $P_{i}$.

Let $\lambda_{i}(p):=f_{i}\left(p_{1}, \ldots, p_{n}\right) \in L$ denote the winnings for $P_{i}$ for the situation where $P_{j}$ choose strategy $p_{j}(j=1, \ldots, n)$. Thus, if all players $P_{j}(j \neq i)$ choose strategy $g_{j} \in K_{j}$ then $P_{i}$ maximizes his winnigs by choosing $p_{i}$. Then the game has a global equilibrium object if and only if $(\mathrm{Ge})$ holds.

Further, in the case $L:=\mathbb{R}$, as an immediate consequence of the preceding global $n$-person games, we obtain well-known main theorem for $n$-person games of $\mathrm{N}$ as h [1952].

We notice that Nash's equilibrium point is only a case of the global equilibrium object for global $n$-person games.

Roots of algebraic equations as global polynomial equilibrium objects. We note that, by the application of $k$-optimal strategy one can simultaneously obtain the upper and lower bounds of the roots of the following equation

$$
\begin{gathered}
x^{n}=a_{1} x^{n-1}+a_{2} x^{n-2}+\cdots+a_{n} \\
\left(a_{1}+\cdots+a_{n}>0 ; a_{i} \geq 0 \quad(i=1, \ldots, n)\right) .
\end{gathered}
$$

As an immediate consequence of $(\mathrm{G})$ and $(\mathrm{Ge})$ we obtain the following statement de facto, for transversal objects.

Transversal objects. A point $\xi \in \mathbb{R}_{+}:=(0,+\infty)$ is the root (as a transversal object) of the equation $(\mathrm{AE})$ if and only if the following equality holds

$$
\begin{aligned}
\xi & :=\max _{\lambda_{2}, \ldots, \lambda_{n} \in \mathbb{R}_{+}} \min \left\{\lambda_{2}, \ldots, \lambda_{n}, a_{1}+\frac{a_{2}}{\lambda_{2}}+\cdots+\frac{a_{n}}{\left.\lambda_{n}^{n-1}\right\}}=\right. \\
& =\min _{\lambda_{2}, \ldots, \lambda_{n} \in \mathbb{R}_{+}} \max \left\{\lambda_{2}, \ldots, \lambda_{n}, a_{1}+\frac{a_{2}}{\lambda_{2}}+\cdots+\frac{a_{n}}{\lambda_{n}^{n-1}}\right\} .
\end{aligned}
$$

In connection with this, the object (point) $\xi \in \mathbb{R}$ in (Ro) is called a global polynomial equilibrium object (point) of the polynomial game (AE).

Interpretations for (Ro). We can consider $n$-players $P_{1}, \ldots, P_{n}$. Let each player have available a strategy set consisting of a nonempty set $X_{i} \subset \mathbb{R}(i=1, \ldots, n)$. We may regard the components of $a_{i} \in X_{i}(i=1, \ldots, n)$ for suitable $X_{i}$ as for the choices of $P_{i}$. The mappings

$$
f_{i}(x):=x^{i}+a_{1} x^{i-1}+\cdots+a_{i}: \mathbb{R} \rightarrow \mathbb{R}
$$

are continuous and represents the loss of $P_{i}$ if each player $P_{j}$ chooses the strategy $a_{j} \in X_{j}$. Then there exists a global polynomial equilibrium point $\xi \in \mathbb{R}$ such that (Ro). In this game we have that $g\left(\lambda_{1}, \ldots, \lambda_{n}\right):=a_{1}+a_{2} / \lambda_{2}+\cdots+a_{n} / \lambda_{n}^{n-1}$ !

Applying $k$-optimal strategy we obtain directly the following equality for positive root of equation (AE), i.e., for the following transversal sets. Thus we have a new economicaly $n$-person game.

Theorem 12.1. (Polynomial $n$-person game). Let $I_{1}, \ldots, I_{n}$ be indices sets and $\theta_{i_{j}} \geq 0$ be real numbers which satisfy the following condition

$$
\sum_{i_{j} \in I_{j}} \theta_{i_{j}}=j-t \quad \text { for } j=1, \ldots, n \text { and } 0<t<1 .
$$

Then $\zeta \in \mathbb{R}_{+}$is the root (as a global polynomial equilibrium point) of the following algebraic equation of the form $x^{t}=a_{1} x^{t-1}+\cdots+a_{n} x^{t-n}\left(\left(a_{1}, \ldots, a_{n}\right) \neq(0, \ldots, 0)\right)$ if and only if the following equality holds

$$
\max _{M_{i_{j}}} \min \left\{M_{i_{j}},\left(\sum_{j=1}^{n} \frac{a_{j}}{\prod_{i_{j} \in I_{j}} M_{i_{j}}^{\theta_{i_{j}}}}\right)^{1 / t}\right\}=\min _{M_{i_{j}}} \max \left\{M_{i_{j}},\left(\sum_{j=1}^{n} \frac{a_{j}}{\prod_{i_{j} \in I_{j}} M_{i_{j}}^{\theta_{i_{j}}}}\right)^{1 / t}\right\}:=\zeta .
$$


Interpretations for polynomial $n$-person games. Further, we can consider $n$-players $P_{1}, \ldots, P_{n}$. Let each player have available a strategy set consisting of a nonempty set $X_{i} \subset \mathbb{R}$ $(i=1, \ldots, n)$. We may regard the components of $a_{i} \in X_{i}(i=1, \ldots, n)$ for suitable $X_{i}$ as for the choices of $P_{i}$. The maps

$$
f_{i}(x):=x^{t}+a_{1} x^{t-1}+\cdots+a_{i} x^{t-i} \quad\left(\left(a_{1}, \ldots, a_{i}\right) \neq(0, \ldots, 0)\right)
$$

are continuous and represents the loss of $P_{i}$ if each player $P_{j}$ chooses the strategy $a_{j} \in X_{j}$. Then there exists a polynomial equilibrium point $\xi \in \mathbb{R}$ such that $(\mathrm{Pe})$. In this polynomial $n$-person game we have that

$$
g\left(M_{i_{1}}, \ldots, M_{i_{n}}\right):=\left(\sum_{j=1}^{n} \frac{a_{j}}{\prod_{i_{j} \in I_{j}} M_{i_{j}}^{\theta_{i_{j}}}}\right)^{1 / t} .
$$

Transversal $n$-person games. We consider $n$-players $P_{1}, \ldots, P_{n}$. Let each player have available a strategy set consisting of a nonempty compact, convex set $K_{i}$ in a topological vector space $E_{i}$ for all $i=1, \ldots, n$. Then, for a bisection (controlling) function $g: \mathbb{R}^{n} \rightarrow \mathbb{R}$, there exist at least countable or finite transversal equilibrium elements $\xi_{k} \in \mathbb{R}$ in the following form as

$$
\begin{aligned}
\xi_{k} & :=\max _{\lambda_{1}, \ldots, \lambda_{n} \in \mathbb{R}} \min \left\{\lambda_{1}, \ldots, \lambda_{n}, g\left(\lambda_{1}, \ldots, \lambda_{n}\right)\right\}= \\
& =\min _{\lambda_{1}, \ldots, \lambda_{n} \in \mathbb{R}} \max \left\{\lambda_{1}, \ldots, \lambda_{n}, g\left(\lambda_{1}, \ldots, \lambda_{n}\right)\right\}
\end{aligned}
$$

if in addition the following facts are satisfied: 1) all maps $\lambda_{i}: K_{1} \times \cdots \times K_{n} \rightarrow$ $\mathbb{R}$ are continuous and 2) all maps $p \mapsto \lambda_{i}(p)$ are convex (if we fix an arbitrary $p_{i} \in K_{i}$ in $\left.p=\left(p_{1}, \ldots, p_{n}\right)\right)$.

Annotations. The number $\xi_{k} \in \mathbb{R}$ represents the loss of $P_{i}$ if each player $P_{j}$ chooses the strategy $p_{j} \in K_{j}$. If

$$
g\left(\lambda_{1}, \ldots, \lambda_{n}\right):=\min _{p_{i} \in K_{i}} \lambda_{i}\left(q_{1}, \ldots, q_{i-1}, p_{i}, q_{i+1}, \ldots, q_{n}\right)
$$

then we obtain well-known Nash's $n$-person game. The function $g: \mathbb{R}^{n} \rightarrow$ $\mathbb{R}$ is a controlling function. In a transversal equilibrium point no player has a reason to change his strategy in the other players keep their strategy (loss minimization). The following facts are essential as direct consequences of the Axiom of Infinite Choice and Theorem 6.11:

1) Every transversal n-person game has at least countable or finite solutions $(=$ : transversal equilibrium elements).

2) Every Nash's n-person game has at least countable or finite solutions (=: Nash's equilibrium points).

\section{Alternative $n$-Person Games}

By a multivalued map or a correspodence $T: M \subset X \rightarrow 2^{Y}$ we mean a map which assings to each point $x \in M$ a subset $T(x) \subset Y$. For $M=\varnothing$, let $T=\varnothing$. Here $2^{Y}$ denotes the set of all subsets of $Y$. Every singlevalued map $R: X \rightarrow Y$ can be identified with a multivalued map of the preceding 
form by setting $T(x)=\{R(x)\}$ for all $x \in X$. Thus $T(x)$ is a singleton, consisting of the image point $R(x)$.

The point $\xi$ is called a fixed point of $T$ iff $\xi \in T(\xi)$. Naturally, $T(M)$ denotes the union of all sets $T(x)$ over $x \in M$, i.e.,

$$
T(M)=\bigcup_{X \in M} T(x) .
$$

The graph of $T$ is defined to be the set $G(M)=\{(x, y): x \in M, y \in T(x)\}$. An important example of a multivalued map is the solution operator $T$ of the equation $S(y)=x$ and $S: Y \rightarrow X$, which assings to each point $x \in X$ the set $T(x)$ of solutions $y \in Y$. If there is no solution, then $T(x)=\varnothing$. This $T$ is called the generalized inverse operator of $S$. The theory of multivalued maps has the important applications in game theory and mathematical exonomics.

Further, let $T: M \rightarrow 2^{N}$ be a multivalued map, where $M$ and $N$ are topological spaces. If $A \subset N$, the preimage $T^{-1}(A)$ is defined maturally enough to be the set of all $x \in M$ with $T(x) \cap A \neq \varnothing$. The map $T$ is called upper semicontinuous iff $T^{-1}(A)$ is closed for all closed sets $A$ in $N$. The map $T$ is called lower semicontinuous iff $T^{-1}(A)$ is open for all open sets $A$ in $N$.

Annotatons. For a single valued map $T: M \rightarrow N$ upper semicontinuous and lower semicontinuous is identical with sontinuous. If $M \subset X$ and $N \subset Y$, where $X$ and $Y$ are Banach spaces or more generally, locally convex spaces, then $M$ and $N$ are to have the induced toplogy. Open and closed in definitions then means relatively open and relatively closed, respectively. For closed subsets $M$ and $N$, however relatively closed and closed in $X$ and $Y$ are the same. Similarly, for open subsets $M$ and $N$, relatively open and open in $X$ and $Y$ are the same.

Under the preceding conditions, the map $T$ is upper semicontinuous if and only of for every $x \in M$ and every open set $V$ in $N$ with $T(x) \in V$, there exists a neighborhood $U(x)$ such that $T(U(x)) \subset V$.

Also, the map $T$ is lower semicontinuous if and only if for every $x \in M$ and every neighborhood $V(y)$ of every $y \in T(x)$, there exists a neighborhood $U(x)$ such that $T(u) \cap V(y) \neq \varnothing$ for all $u \in U(x)$.

Proposition 13.1. Let $X$ be a locally convex space and let $K$ be a nonempty compact convex subset of $X$. If $T: K \rightarrow 2^{K}$ if the set $T(x)$ is nonempty convex for all $x \in K$ and the preimages $T^{-1}(y)$ are relatively open with respect to $K$ for all $y \in K$, then $T$ has at least countable or finite fixed points.

Proof. We regard $X$ as a real space. Since $K$ is compact and the set $T^{-1}(y)$ is open in $K$, there are finitely many points $y_{1}, \ldots, y_{n}$ such that

$$
K=\bigcup_{i=1}^{n} T^{-1}\left(y_{i}\right) .
$$

Let $\left\{f_{1}, \ldots, f_{n}\right\}$ be a partition of unity subordinate to this cover. Set in the following that is $K_{0}=C l\left(\operatorname{conv}\left\{y_{1}, \ldots, y_{n}\right\}\right)$ and

$$
p(x)=\sum_{i=1}^{n} f_{i}(x) y_{i} \quad \text { for all } \quad x \in K_{0} ;
$$

hence, if $X_{0}=\operatorname{span}\left(\left\{y_{1}, \ldots, y_{n}\right\}\right)$, then $K_{0} \subset X_{0}$ and $\operatorname{diam}\left(X_{0}\right)<\infty$. The mapping $p$ is continuous and $p\left(K_{0}\right) \subset K_{0}$. For $f_{i}(i=1, \ldots, n)$ is continuous and for all $x \in K_{0}$ we have $0 \leq f_{i}(x) \leq 1$ and $\sum_{i=1}^{n} f_{i}(x)=1$. We show that $p(x) \in T(x)$ for all $x \in K_{0}$. Indeed, if $f_{i}(x) \neq 0$, then the fact that $\sup \left(f_{i}\right) \subset T^{-1}\left(y_{i}\right)$ immediately implies that $x \in T^{-1}\left(y_{i}\right)$, so that $y_{i} \in T(x)$ and $T(x)$ is convex. 
Applyng Theorem 6.7 to the mapping $p: K_{0} \rightarrow K_{0}$ provides us with the existence at least countable or finite fixed points $x_{k}=p\left(x_{k}\right)$, i.e., $x_{k} \in T\left(x_{k}\right)$. The proof is complete.

Variational Inequalities. In this section we apply some forms statements of Generalized Brouwer fixed point theorem for the proof of the following Hartman-Stampacchia variational inequality in the form as:

$$
\left\langle T\left(x_{0}\right), x_{0}-x\right\rangle \geq 0 \text { for all } x \in K \text {. }
$$

Theorem 13.1. Let $K$ be a nonempty, compact and convex set. If the map $T: K \subset X \rightarrow X^{*}$ is continuous, where $X$ is a locally convex space and $X^{*}$ is the corresponding dual space with the strong* topology, then the variational inequality ( $\mathrm{Va}$ ) has at least countable or finite solutions $x_{k} \in K$.

We notice that if $X$ is a Banach space, then the strong* topology on $X^{*}$ is the same as the usual norm topology on $X^{*}$.

Proof of Theorem 13.1 If (Va) is false, then for every $x_{0} \in K$ there is an $x \in K$ such that the following inequality holds as $\left\langle T\left(x_{0}\right), x_{0}-x\right\rangle<0$. We define the multivalued mapping $S: K \rightarrow 2^{K}$ by

$$
S\left(x_{0}\right):=\left\{x \in K:\left\langle T\left(x_{0}\right), x_{0}-x\right\rangle<0\right\} ;
$$

thus, the set $S\left(x_{0}\right)$ is nonempty for all $x_{0} \in K$. Furthermore, $S\left(x_{0}\right)$ is convex. We show that the set $S^{-1}(x)$ is relatively open in $K$. First of all, we have

$$
S^{-1}(x)=\left\{x_{0} \in K:\left\langle T\left(x_{0}\right), x_{0}-x\right\rangle<0\right\},
$$

hence, let $\left\{x_{\alpha}\right\}_{\alpha \in I}$ be a Moore-Smith sequence in $K \backslash S^{-1}(x)$ with $x_{\alpha} \rightarrow y$, so that $\left\langle T\left(x_{\alpha}\right), x_{\alpha}-x\right\rangle \geq 0$ for all $\alpha \in I$. Since $T$ is continuous, $T\left(x_{\alpha}\right)$ converges to $T(y)$ in the strong* topology, so $\langle T(y), y-x\rangle \geq 0$, and $y \in K \backslash S^{-1}(x)$.

Thus $K \backslash S^{-1}(x)$ is relatively closed and $S^{-1}(x)$ is relatively open. By Proposition 13.1 there exists at least countable or finite fixed points $x_{k} \in$ $S\left(x_{k}\right)$. This leads to the contradiction $\left\langle T\left(x_{k}\right), x_{k}-x_{k}\right\rangle<0$. The proof is complete.

Our goal is to give Browder Fixed Point Theorem for multivalued maps with boundary conditions. It is the following result.

Theorem 13.2. (Browder [1968], Tasković [2005]). Let $K$ be a nonempty compact convex set in a locally convex space $X$. If the map $T: K \rightarrow 2^{X}$ is upper semicontinuous, if the set $T(x)$ is nonempty closed and convex for all $x \in K$ such that one of the following two boundary conditions is satisfied: (1') for every $x \in \partial K$ there are points $y \in T(x)$ and $u \in K$, and a number $\lambda>0$ such that $y=x+\lambda(u-x)$; (2') for every $x \in \partial K$ there are points $y \in T(x)$ and $u \in K$, and a number $\lambda<0$ such that $y=x+\lambda(u-x)$. Then $T$ has at least countable or finite fixed points.

Proof. We assume that (1') holds and that $T$ has no fixed point. Let $M(x)=\{x\} \backslash T(x)$ for all $x \in K$. THe set $M(x)$ is closed, convex, and 
nonempty. Since $0 \ni M(x)$, Hahn-Banach theorem for convex sets says that there is a functional $w(x) \in X^{*}$ such that $(w(x), z)<0$ for all $z \in M(x)$. By choosing a suitable cover of $M(x)$, we can find an open set $U(M(x))$ such that $M(x) \subset U(M(x))$ and $(w(x), z)<0$ for all $\in U(M(x))$. We define

$$
N(w(x))=\{v \in K:(w(x), z)<0 \text { for all } z \in M(v)\},
$$

the map $T$ is upper semicontinuous, so that for every $x_{i} \in K$ there exists a neighborhood $V\left(x_{i}\right)$, relative open in $K$, such that $M(v) \subset U\left(M\left(x_{i}\right)\right)$ for all $v \in V\left(x_{i}\right)$. Thus $V\left(x_{i}\right) \subset N\left(w\left(x_{i}\right)\right)$. The set $K$ is compact, and hence covered by finitely many of the $V\left(x_{i}\right)$, so $K=\bigcup_{i=1}^{n} V\left(x_{i}\right)$. Let

$$
r(x)=\sum_{i=1}^{n} f_{i}(x) w\left(x_{i}\right) \quad \text { for all } x \in K,
$$

where $\left\{f_{1}, \ldots, f-N\right\}$ is a partition of unity subordinate to this cover. The map $r: K \rightarrow X^{*}$ is continuous whenever $X^{*}$ has the strong* topology. By Theorem 13.1 there axist at least countable or finite $x \in K$ such that $(r(x), x-v) \geq 0$ for all $v \in K$. Let $x \in \operatorname{int}(K)$. For every $z \in X$ there is then an $\varepsilon>0$ such that $x \pm \varepsilon z \in K$. From the preceding facts $\mp(r(x), z) \geq 0$ for all $z \in X$, which says that $r(x)=0$ in contradicion with $(r(x), z)<0$ for all $z \in M(x)$. Also, for $x \in \partial K$, by (1') there exists $y \in T(x)$ such that $(r(x), x-y)<0$ for $x-y \in M(x)$. This again contradicts $(r(x), x-y) \geq 0$. This proves statement in the case $\left(1^{\prime}\right)$. For the case of boundary condition $(2$ '), simple replace the " $<"$ sign with the " $>$ " sign we have the proof, also. The proof is complete.

Theorem 13.3. (Generalized Kakutani theorem). Let $K$ be a nonempty compact convex set in a locally convex space $X$, let the set $T(x)$ be nonempty closed and convex for all $x \in K$, and let the multivalued map $T: K \rightarrow 2^{K}$ be upper semicontuous, then $T$ has at least countable or finite fixed points.

Observe that Kakutani fixed point theorem (Theorem 7.3) as and the preceding Generalized Theorem of Kakutani (Theorem 13.3) are special cases of Theorem 13.2. In the case of Theorem 13.3, we have $T(x) \subset K$. Therefore we can choose the point $u=y$ for a fixed $y \in T(x)$, and $\lambda=1$. For the proof see: Tasković [2001, p. 443].

We notice that Kakutani proved this statement for $X=\mathbb{R}^{n}$. The generalization is due to Ky Fan [1952] and Glicksberg [1952]. Newertheles we will look at some important special cases of it here. In this sense, Tychonoff Fixed Point Theorem it follows directly from Theorem 13.3, as and the following two consequences:

Corollary 13.1. (Generalized Theorem of Tychonoff). Let $T: K \subset X \rightarrow$ $K$ be continuous, where $K$ is a nonempthy, compact, convex set in locally convex space $X$. Then $T$ has at least countable or finite fixed points.

Corollary 13.2. (Global Schauder Fixed Point Theorem, Tasković [2005]). Let $M$ be a nonempty closed bounded and convex subset in the reflexive separable Banach space $X$. If the map $T: M \rightarrow M$ is weakly sequentally continuous (i.e., if $x_{n} \rightarrow x$ as $n \rightarrow \infty$, then also $T\left(x_{n}\right) \rightarrow T(x)$ ), then $T$ has at least countable or finite fixed points. 
Proof. Let $X$ have the weak topology. Then $X$ becomes a locally convex space. The set $M$ is weakly compact. Since $X$ is separable, the weak topology on $M$ is metrizable. Therefore $T$ is weakly continuous. Now the conclusion follows from Generalized Tychonoff Fixed Point Theorem.

Corollary 13.3. (Tasković, [2005]). Let $M$ be a nonempty closed convex set in the Banach space $X, T: M \rightarrow 2^{M}$ is upper semicontinuous, $T(M)$ is relatively compact, and the set $T(x)$ is nonempty closed and convex for all $x \in M$, then $T$ has at least countable or finite fixed points.

Proof. Set $K=C l(\operatorname{conv}(T(M)))$, so $K \subset M$, and aplly Theorem 13.3 to the restriction $T: K \rightarrow 2^{K}$. The proof is complete.

Transversal reasonable pricing systems. We consider an economic system with $n$ producers $P_{1}, \ldots, P_{n}$, who simultaneously produce goods and sell them to other prodicers. Let $P_{i}$ produce $a_{i}$ goods $G_{i}$ per unit of time (e.g., per month). Let $p_{i}$ be the price for one $G_{i}$. The proces are to be variable. We want to find a reasonable pricing system, $p=\left(p_{1}, \ldots, p_{n}\right)$. For the end, we assume that we have the following transversal represents control (or function control) $p \mapsto g\left(p_{1}, \ldots, p_{n}\right)$ as an interpretation of some nature fact (for example, as an economic interpretation). In the case for function control in the form

$$
g\left(p_{1}, \ldots, p_{n}\right):=p_{i} a_{i}=\sum_{j=1}^{n} p_{j} D_{i j}(p),
$$

and $D_{i j}(p) \equiv 0$ for $i=1, \ldots, n$ we have the following economic interpretation: The number $D_{i j}(p)$ is the demand by produces $P_{i}$ for good $G_{i}$. Thus $p_{j} D_{i j}$ is equal to the price which $P_{i}$ must pay for $G_{j}$ if $P_{i}$ wishes to purchase the desired amount. Further $p_{i} a_{i}$ is equal to the income which $P_{i}$ realizes from the sale of $G_{i}$. Now (Fc) says that all procedure use all of their income to obtain more goods. Let $p>0$ mean that $p_{i} \geq 0$ for all $i$ and $p \neq 0$.

Use Theorem 13.3 (Generalized Kakutani theorem) we have following result: If the nonnegative continuous functions $p \mapsto D_{i j}(p)$ satidfy $(F c)$ for all $p>0$, and if the given fixed numbers $a_{i}$ are all positive, then there exist at least countable or finite pricing systems $p_{k}>0$ such that

$$
a_{j}-\sum_{i=1}^{n} D_{i j}\left(P_{k}\right) \geq 0, \quad j=1, \ldots, n,
$$

furthemore, of the sign " $>$ " holds in this inequality for some $r$, then $p_{r}=0$.

Interpretation. The economic interpretation of these results is as follows. There is at least countable or finite pricing systems for which the supple are at least as great as the demand. The price of goods in excess supply is zero.

Existence of a transversal reasonable pricing system: In connection with the preceding, if $p_{i}: X \rightarrow \mathbb{R}(i=1, \ldots, n)$ and $X$ is a the function control $p \mapsto g\left(p_{1}, \ldots, p_{n}\right)$ is a decreasing function such that

$$
p_{1}\left(b_{1}\right)=\cdots=p_{n}\left(b_{n}\right):=\xi=g(\xi, \ldots, \xi)
$$

for some $b_{1}, \ldots, b_{n} \in X$ if and only if the following equality holds in the form as

$$
\xi:=\min _{p_{1}, \ldots, p_{n} \in \mathbb{R}} \max \left\{p_{1}, \ldots, p_{n}, g\left(p_{1}, \ldots, p_{n}\right)\right\}=
$$




$$
=\max _{p_{1}, \ldots, p_{n} \in \mathbb{R}} \min \left\{p_{1}, \ldots, p_{n}, g\left(p_{1}, \ldots, p_{n}\right)\right\} .
$$

This result follows direct from the facts in Tasković [2005]. The object (point) $\xi \in \mathbb{R}$ in this equality is called global equilibrium pricing of the global princing system game.

Interpretation: There is a solution $\xi \in \mathbb{R}$ of the global pricing system game for which the supply is at least as great as the demaned.

Theorem 13.4. (Global alternative $n$-person game). If $L:=(L, \preccurlyeq)$ is a complete lattice with the local comparability and if $g: L^{n} \rightarrow L(n \in \mathbb{N}$ is a fixed number) is a decreasing mapping, then there exist at least countable or finite global equilibrium objects $\xi_{k} \in L$.

This statement is an immediate consequence of the facts $(\mathrm{G})$ and $(\mathrm{Ge})$ and the well-konwn fact: that every decreasing mapping on complete lattice has at least countable or finite fixed apices.

Further, adequate with these facts, use Theorem 6.7 (General Brouwer theorem) we obtain direct the following solutions for the global $n$-person games.

Theorem 13.5. (General $n$-person game). Let $K_{i}$ be a compact convex nonempty set in a topological vector space $E_{i}$ for all $i=1, \ldots, n$. If $g_{i}$ : $\mathbb{R}^{n} \rightarrow \mathbb{R}$ defined as $g_{i}(p):=g_{i}\left(\lambda_{1}\left(p_{1}\right), \ldots, \lambda_{n}\left(p_{n}\right)\right)$ and $\lambda_{i}\left(p_{i}\right): K_{i} \rightarrow \mathbb{R}$, are continuous for $i=1, \ldots, n$, then there exist at least countable or finite global equilibrium objects $\xi_{k} \in \mathbb{R}$.

Interpretations. We consider $n$-players $P_{1}, \ldots, P_{n}$. Let each player have available a trategy set sonsisting of a nonempty set $K_{i} \subset E_{i}(i=1, \ldots, n)$. We may regard the components of $p_{i} \in K_{i}(i=1, \ldots, n)$ for suitable $K_{i}$ as for the choices of $P_{i}$.

Let $\lambda_{i}(p):=f_{i}\left(p_{1}, \ldots, p_{n}\right) \in \mathbb{R}$ denote the winnings for $P_{i}$ for the situation where $P_{j}$ choses strategy $P_{j}(j=1, \ldots, n)$. Thus, if all players $P_{j}(j \neq i)$ choose strategy $q_{j} \in K_{j}$, then $P_{i}$ maximizes his winnings by choosing $p_{i}$. Then the global n-person game has at least countable or finite global equilibrium objects (points) $\xi_{k} \in \mathbb{R}$.

Further, as an immediate consequence of the preceding general $n$-person games, we obtain well-known main theorem for $n$-person games of $\mathrm{N}$ ash [1951].

Main theorem for $n$-person games of Nash. Now we consider $n$-players $P_{1}, \ldots, P_{n}$. Supose, each player $P_{i}$ has a strategy set $K_{i}$ available. We assume: $K_{i}$ is a compact, convex, nonempty set in a topological vector space $E_{i}$ for all $i=1, \ldots, n$. The maps $f_{i}: K_{1} \times \cdots \times K_{n} \rightarrow \mathbb{R}$ are continuous, represents the loss of $P_{i}$ if each player $P_{j}$ chooses the strategy $p_{j} \in K_{j}$, and all maps $p \mapsto f(p)$ are convex if we fix an arbitrary $p_{i}$ in $p=\left(p_{1}, \ldots, p_{n}\right)$. Then there exists a Nash equilibrium point, i.e., there exists a point $\left(q_{1}, \ldots, q_{n}\right)$ with $q_{j} \in K_{j}$ for all $j=1, \ldots, n$ such that for all $i=1, \ldots, n$

$$
f_{i}\left(q_{1}, \ldots, q_{n}\right)=\min _{p_{i} \in K_{i}} f_{i}\left(q_{1}, \ldots, q_{i-1}, p_{i}, q_{i+1}, \ldots, q_{n}\right) .
$$

Moreover, from Theorem 13.5 it follows that every $n$-person game has at least countable or finite Nash equilibrium points!

Indeed, let the function control $g\left(f_{1}, \ldots, f_{n}\right):=f_{i}\left(p_{1}, \ldots, p_{n}\right)$ for $i=1, \ldots, n$. Since the equation $f_{i}(\bar{p})=\min _{t_{i} \in K_{i}} f_{i}(\bar{p})$ for $\bar{p}:=\left(q_{1}, \ldots, q_{i-1}, p_{i}, q_{i+1}, \ldots, q_{n}\right)$ has at least one solution (as the continuous function $f_{i}$ on the compact set $K_{i}$ ), it follows that then hold all conditions in Theorem 13.5, thus applying the preceding general $n$-person game to this $n$-person game we obtain that there is at least countable or finite Nash equilibrium points of the game.

Annotation. We notice taht this fact has a simple interpretation. In a Nash equilibrium point no player has a reason to change his strategy if the other players keep their strategy (loss 
minimization)! Also, if $E_{i}=\mathbb{R}^{n_{i}}$ and

$$
K_{i}:=\left\{p_{i} \in E_{i}: 0 \leq p_{i, k} \leq 1 ; k=1, \ldots, n_{i}\right\},
$$

then the $p_{i, k}$ can be viewed as the probability with which player $P_{i}$ makes his $k$ th decision.

Further, we obtained an alternative minimax statement by using a fixed point statement for multivalued maps.

Theorem 13.6. (Tasković, [2012]). Suppose that $A$ and $B$ is a nonempty, closed, bounded, convex set in a reflexive Banach space $X$ and $Y$ respectively. Let $f: A \times B \rightarrow \mathbb{R}$ be a function such that $x \mapsto f(x, y)$ is lower semicontinuous and quasi-convex on $A$ for all $y \in B$, and $y \mapsto f(x, y)$ is upper semicontinuous and quasi-concave on $B$ for all $x \in A$. Then the function $f$ has at least countable or finite saddle points $\left(x_{k}, y_{k}\right)$ such that

$$
\min _{x \in A} \max _{y \in B} f(x, y)=\max _{y \in B} \min _{x \in A} f(x, y) \equiv f\left(x_{k}, y_{k}\right) .
$$

Proof. (Application of Proposition 13.1). We set $a=\min _{x \in A} \max _{y \in B} f(x, y)$ and we set that $b=\max _{y \in B} \min _{x \in A} f(x, y)$. First, we show that $b$ exists. By the equality (9), there is, for every $y \in B$ a point $z(y) \in A$ with

$$
f(z(y), y)=\min _{x \in A} f(x, y) .
$$

We set $h(y)=-f(z(y), y)$. Then we have $-f(x, y) \leq h(y)$ for all $x \in$ $A$. We show below that the function $h: B \rightarrow \mathbb{R}$ is lower continuous and quasiconvex. Therefore $b=-\min _{y \in B} h(y)$ exists. We set $A_{t}=\{y \in B$ : $h(y) \leq t\}$ and

$$
B_{t}(w)=\{y \in B:-f(z(w), y) \leq t\} ;
$$

then for $t \in \mathbb{R}$ fixed, it follows that $A_{t} \subset B_{t}(w)$ for all $w \in B$. Hence, $B_{t}(w)$ is closed and convex.

We show that $h$ is lower semicontinuous, i.e., the set $A_{t}$ is closed for all $t \in \mathbb{R}$. Indeed, if $\left(y_{n}\right)$ is any sequence in $A_{t}$ with $y_{n} \rightarrow w$ as $n \rightarrow \infty$, then $y_{n} \in B_{t}(w)$. Hence $w \in B_{t}(w)$, so that $h(w) \leq t$. Thus $w \in A_{t}$.

We show that $h$ is quasi-convex, i.e., $A_{t}$ is convex for all $t \in \mathbb{R}$. Indeed, suppose $v, y \in A_{t}$. Set $w=\lambda v+(1-\lambda) y$ for $0 \leq \lambda \leq 1$. Then $v, y \in B_{t}(w)$ and hence $w \in B_{t}(w)$, so $w \in A_{t}$. We notice that existence of $a$ is established similarly. The inequality $b \leq a$ follows immediately from

$$
\min _{x \in A} f(x, y) \leq \min _{x \in A} \max _{y \in B} f(x, y)=a .
$$

We show that $a \leq b$ with the aid of the fixed-point result in Proposition 7. To this end we provide $X$ and $Y$ with the weak topology. Let $s=a-\varepsilon$ and $t=b+\varepsilon$ for $\varepsilon>0$. We construct a map $T: A \times B \rightarrow 2^{A \times B}$ by setting

$$
T(x, y)=\{(u, v) \in A \times B: f(u, y)<t, f(x, v)>s\} .
$$


That $T(x, y) \neq \varnothing$ follows from $\min _{x \in A} f(x, y) \leq b$ and $\max _{y \in B} f(x, y) \geq$ $a$. The set $T(x, y)$ is convex, by conditions of statement. On the other hand, the preimage

$$
T^{-1}(u, v)=\{(x, y) \in A \times B: f(u, y)<t, f(x, v)>s\}
$$

is weakly relatively open in $A \times B$. For the sets in the following adequate forms as

$$
\{x \in A: f(x, v) \leq s\} \quad \text { and } \quad\{y \in B: f(u, y) \geq t\}
$$

are closed and convex by the conditions of statement, and hence are weakly closed with respect to $A, B$. Therefore the complementary sets

$$
\{x \in A: f(x, v)>s\} \quad \text { and } \quad\{y \in B: f(x, y)<t\}
$$

are weakly open. By Proposition 13.1, there is at least countable or finite fixed points of $T$, i.e., $(x, y) \in T(x, y)$. Thus we obtain the following inequalities in the form as

$$
a-\varepsilon=s<f(x, y)<t=b+\varepsilon,
$$

and since $\varepsilon>0$ is arbitrary, we have that $a \leq b$. Thus $a=b$ and the conclusion follows from the definition that $\left(x_{k}, y_{k}\right) \in A \times B$ is a saddle point of $f$ iff

$$
f\left(x_{k}, y\right) \leq f\left(x_{k}, y_{k}\right) \leq f\left(x, y_{k}\right)
$$

for all $(x, y) \in A \times B$. The proof is complete.

\section{Peano theorem For inclusions}

Such differential inclusions are mathematical models of dynamical systems for which we have no complete description. We consider the initial value problem

$$
x^{\prime}(x) \in F(x(t), t), \quad x\left(t_{0}\right)=x_{0},
$$

where $t \in \mathbb{R}$ and $x \in \mathbb{R}^{n}$. Here, $F(x, t)$ associates to the state $x$ of the system at time $t$, the set of feasible velocities. Examples for ths are given by large system in biology, economics, and the social scienci. We are looking for a solution $x: \mathbb{R} \rightarrow \mathbb{R}^{n}$.

Theorem 14.1. (Generalized Peano Theorem). We are given $t_{0} \in \mathbb{R}$ and $x_{0} \in \mathbb{R}^{n}$, where $\left(x_{0}, t_{0}\right) \in U$. Let $F: U \subset \mathbb{R}^{n+1} \rightarrow 2^{\mathbb{R}^{n}}$ be a lower semicontinuous multivalued map on the region $U$ such that $F(x, t)$ is a nonempty closed convex set in $\mathbb{R}^{n}$ for all $(x, t) \in U$. Then the value problem (Pp) has at least countable or finite $C^{1}$-solutions $x=x(t)$ in a neighborhood of $t_{0}$.

Proof. By Michael's selection theorem there is a continuous selection $f$ : $U \rightarrow \mathbb{R}^{n}$ of $F$. So it is sufficient to consider the classical initaial value problem

$$
x^{\prime}(t)=f(x(t), t), \quad x\left(t_{0}\right)=x_{0} .
$$


Now the local result (10) follows from the classical Generalized Peano theorem (Proposition 9.1). The proof is complete.

Annotation. As a reference for differential inclusions, we recommedn Aubin-Cellina [1983]. There one also finds interesting applications to optimal control and viability theory.

\section{REFERENCES}

[1] Banach, Stefan. Sur l'équation fonctionnelle, Fund. Math., 1 (1920), 123-Ây124.

[2] Banach, Stefan. Sur les opérations dans les ensembles abstraits et leur application aux équations intégrales, Fund. Math., 3 (1922), 133-181.

[3] Banach, Stefan. Sur les fonctionnelles linéaires I-II, Studia Math., 1 (1929), 211-216 i $223-239$.

[4] Banach, Stefan. Théorie des opérations linéaires, Monografie Matematyczne, t.1, Varsovie, 1932.

[5] Brouwer, L. E. J. On continuous one-to-one transformations of surfaces into themselves, Proc. Kon. Ned. Ak. V. Wet. Ser. A, 11 (1909), 788-798; 12 (1910), 286-297; 13 (1911), 767-777; 14 (1912), 300-310; 15 (1913), 352-360; 22 (1920), 811-Ầ814; 23 (1921), 232-234.

[6] Brouwer, L. E. J. Invarianz des n-dimensionalen Gebiets, Math. Ann., 71 (1912), 305-313; 72 (1912), 55-56.

[7] Brouwer, L. E. J. Über Abbildungen von Mannigfaltigkeiten, Math. Ann., 71 (1912), $97-115$.

[8] Brouwer, L. E. J. An intuitionist cerrection of the fixed point theorem on the sphere, Proc. Roy. Soc. London, (A)-213 (1952), 1-2.

[9] Birkhoff, Garrett. Lattice theory, Amer. Math. Soc. Collog. Publ. vol. 25, New York, 1948, p.p. 285.

[10] Bourbaki, Nicolas. Sur le théorème de Zorn, Archiv der Math., 2 (1950), 434-437.

[11] Dugundji, J., and Granas, Andrzej. Weakly contractive maps and elementary domain invariance theorem, Bull. Greek Math. Soc., 19 (1978), 141-151.

[12] Dugundji, J., and Granas, Andrzej. Fixed point theory, Vol. I., Monografie Mathematyczne 61, Polska Acad. Nauk, 1982, Warsaw, 209 p.p.

[13] Dugundji, James. An extension of Tietze's theorem, Pacific J. Math., 1 (1951), 353367.

[14] Dugundji, James. Absolute neighbourhood retracts and local connectedness in arbitrary metric spaces, Compos. Math., 13 (1958), 229-246.

[15] Dugundji, James. Topology, Allyn and Bacon, Inc., Boston, 1965.

[16] Dugundji, James. Positive definite functions and coincidences, Fundamenta Math., 90 (1976), 131-142.

[17] Fan, Ky. Fixed point and minimax theorems in locally convex linear spaces, Proc. Nat. Acad. Sci. USA, 38 (1952), 121-126. 
[18] Fan, Ky. A generalization of Tucker's combinatorial lemma with topological application, Ann. of Math. 56 (1952), 431-437.

[19] Fan, Ky. Minimax theorems, Proc. Nat. Acad. Sci. USA, 39 (1953), 42-47.

[20] Fan, Ky. Topological proofs for certain theorems on metrices with non-negative elements, Monatsh. Math., 62 (1958), 219-237.

[21] Fan, Ky. A generalization of Tychonoff's fixed point theorem, Math. Ann., 142 (1961), 305-310.

[22] Fan, Ky. Sur un theoreme minimax, C. R. Acad. Sci. Paris, 259 (1964), 3925-3928.

[23] Fan, Ky. Applications of a theorem concerning sets with convex sections, Math. Ann., 163 (1966), 189-203.

[24] Fan, Ky. Extensions of two fixed point theorems of F. E. Browder, Math. Z., 112 (1969), 234-240.

[25] Fan, Ky. A minimax inequality and applications, in: Inequalities, Vol. III, Academic Press, 1972.

[26] Gödel, Kurt. The consistency of the continuum hypothesis, Ann. of Math. Stud., vol. 3, Princeton Univ. Press, Princeton, NJ, 1940, pp. 66.

[27] Howard, Paul., and Rubin, Jean. E. Consequences of the Axiom of Choice, American Math. Society, Vol. 59, 1998. Mathematical Surveys and Monographs, 432 pages.

[28] Jech, T. J. The Axiom of Choice, Amsterdam, North-Holland, 1973.

[29] Kuratowski, C. Une methode d'elimination des nombers transfinies raisonnements mathematiques, Fund. Math. 3 (1922), 76-108.

[30] Leray, Jean. Topologie des espaces abstraits de M. Banach, C. R. Acad. Sci. Paris, 200 (1935), 1083-1093.

[31] Leray, Jean. Majoration des dérivées secondes des solutions d'un probléme de Dirichlet, J. Math. Pures Appl., 17 (1938), 89-104.

[32] Leray, Jean. Sur la forme des espaces topologiques et sur les points fixés des représentations, J. Math. Pures Appl., 24 (1945), 95-167.

[33] Moore, Gregory. H. Zermelo's Axiom of Choice $\hat{a} \breve{A} S$ its origins, development, and influence; Springer-ÂyVerlag, New York, Heidelberg, Berlin, Vol. 8, 1982, p.p. 410.

[34] Nash, J. Non-cooperative games, Ann. of Math., 54 (1951), 286-295.

[35] Nash, J. The embedding problem for Riemann manifolds, Ann. of Math., 63 (1956), 20-63.

[36] Nash, J. Continuity of solutions of parabolic and eliptic equations, Amer. J. Math., 80 (1958), 931-954.

[37] Neumann, J. (Von Neumann, John.). Zur Theorie der Gesellschaftsspiele, Math. Annalen, 100 (1928), 295-320.

[38] Neumann, J. (Von Neumann, John.). Mathematische Grundlagen der Quantenmechanik, Springer-Verlag, Berlin 1932. (English edition: Mathematical Foundations of Quantum Mechanics, University Press, Princeton, NJ 1932. 
[39] Neumann, J. (Von Neumann, John.). On complete topological spaces, Trans. Amer. Math. Soc., 37 (1935), 1-20.

[40] Neumann, J. (Von Neumann, John.). Über ein ökonomisches Gleichungssystem und eine Verallgemeinerung des Brouwerschen Fixpunktsatzes, Erg. Math. Kolloqu., 8 (1937), 73-83.

[41] Rubin, H., and Rubin, J. Equivalents of the Axiom of Choice, North-Holland Publ. Comp., Amsterdam-ÂyLondon, 1970, 134 p.p.;

[42] Rubin, H., and Rubin, J. Equivalents of the Axiom of Choice-II, North-Holland, Publ. Comp. Amsterdam-New York-Oxford, 1985, 322 p.p.

[43] Schauder, Juliusz. Zur Theorie stetiger Abbildungen in Functionalräumen, Math.Z.,26 (1927), 47-65.

[44] Schauder, Juliusz. Bemerkungen zu meiner Arbeit "Zur Theorie stetiger Abbildungen in Functio-nalräumen", Math. Z., 26 (1927), 417-431.

[45] Schauder, Juliusz. Invarianz des Gebietes in Functionalräumen, Studia Math., 1 (1929), 123-139.

[46] Schauder, Juliusz. Der Fixpunktsatz in Functionalräumen, Studia Math., 2 (1930), 171-180.

[47] Stone, Marshall. H. On one parameter unitary gropus in Hilbert space, Ann. of Math., 33 (1932), 643-648.

[48] Stone, Marshall. H. Linear Transformations in Hilbert Space, Amer. Math. Soc., Providence, RI 1932.

[49] Stone, Marshall. H. The theory of representations for Boolean algebras, Trans. Amer. Math. Soc., 40 (1936), 37-111.

[50] Stone, Marshall. H. Applications of the Theory of Boolean Rings to General Topology, Trans. Amer. Math. Soc., 41 (1937), 375-481.

[51] Stone, Marshall. H. Convexity, (Mimeographed lecture notes), The University of Chicago, 1946.

[52] Stone, Marshall. H. The generalized Weierstrass approximation theorem, Mathematics Magazine, 21 (1948), 167-183 and 237-254.

[53] Tarski, Alfred. A lattice theoretical fixpoint theorem and its applications, Pacific. J. Math., 5 (1955), 285-309.

[54] Tasković, Milan. R. Nonlinear Functional Analysis, (Fundamental Elements of Theory). First Book: Monographs, Zavod za udžbenike i nastavna sredstva, Beograd 1993, 792 p.p., (Serbo-Croation). English summary: Comments only new main results of this book, Vol. 1 (1993), 713-752.

[55] Tasković, Milan. R. Nonlinear Functional Analysis, Second Book, Monographs Global Convex Analysis: General convexity, Variational methods and Optimization, Zavod za udžbenike i nastavna sredstva and Vojnoizdavački zavod, Beograd 2001, (In Serbian), 1223 pages. English summary: Transversal and fixed points, forks, general convex functions, and applications, Vol. 2 (2001), 1067-1176. 
[56] Tasković, Milan. R. Theory of transversal point, spaces and forks, Fundamental Elements and Applications, Monographs of a new mathematical theory, VIZ-Beograd 2005, (In Serbian), 1054 pages. English summary: 1001-1022.

[57] Ulam, Stanislaw. Zur Masstheorie in der allgemeinen Mengelehre, Fund. Math., 16 (1930), 140-150.

[58] Zermelo, Ernst. Beweis dass jede Menge wohlgeordnet werden kann, (Aus einem an Herrn Hilbert gerichteten Briefe), Math. Ann., 59 (1904), 514-516.

[59] Neuer Beweis fur die Moglichkeit einer Wohlordnung, Math. Ann. 65 (1908), $107-$ 128; translated in van Heijenoort 1967, 183-198.

[60] Zorn, Max. A remark on method in transfinite algebra, Bull. Amer. Math. Soc. 41 (1935), 667-670.

[61] Zorn, Max. Idempotency of infinite cardinals, Univ. Calif. Publ. in Math., Seminar Reports (Los Angeles), 2 (1944), 9-12.

[62] M. Tasković, On Schauder's 54th Problem in Scottish Book Revisited, Mathematica Moravica, Vol. 6 (2002), 119-126.

Milan R. TASKović

Faculty of Mathematics

P.O. Box 550

11000 BEOGRAD

SERBia

Home address:

Milan R. TAsković

Nehruova 236,

11070 BELGRADE

Serbia

E-mail address: andreja@predrag.us 